\title{
Nonlocal dynamic Kirchhoff plate formulation based on nonlocal operator method
}

\author{
Yongzheng Zhang ${ }^{1}$
}

Received: 12 August 2021 / Accepted: 16 December 2021 / Published online: 25 January 2022

(c) The Author(s) 2022

\begin{abstract}
In this study, we propose a nonlocal operator method (NOM) for the dynamic analysis of (thin) Kirchhoff plates. The nonlocal Hessian operator is derived based on a second-order Taylor series expansion. The NOM does not require any shape functions and associated derivatives as 'classical' approaches such as FEM, drastically facilitating the implementation. Furthermore, NOM is higher order continuous, which is exploited for thin plate analysis that requires $C^{1}$ continuity. The nonlocal dynamic governing formulation and operator energy functional for Kirchhoff plates are derived from a variational principle. The Verlet-velocity algorithm is used for the time discretization. After confirming the accuracy of the nonlocal Hessian operator, several numerical examples are simulated by the nonlocal dynamic Kirchhoff plate formulation.
\end{abstract}

Keywords Nonlocal operator method · Nonlocal Hessian operator · Operator energy functional · Dual-support · Variational principle $\cdot$ Verlet-velocity algorithm

\section{Introduction}

As a common engineering structure, plate/shell is widely used in civil engineering, aerospace and other fields. The mechanical analysis of rectangular thin plates has always been one of the research focuses of scholars and engineers. The governing equation of the Kirchhoff plate bending problem is a fourth-order partial differential equation whose deflection is an independent variable. The numerical method for developing this problem has a wide scientific significance for solving plate/shell problems.

The analysis of Kirchhoff plate bending problems poses challenges to 'classical' finite element formulations [1-5], which are only $C^{0}$ continuous. However, the Kirchhoff plate problem is a fourth-order partial differential equation which requires a $C^{1}$ formulation if weak form based methods such as FEM are employed. An efficient alternative are so-called meshless methods as many of them are higher order continuous. The approximation function of the meshless method [6-11] represented by the Element-free Galerkin method EFG [12-15] is highly smooth, that is, and the high-order

Yongzheng Zhang

yongzheng.zhang@uni-weimar.de

1 Institute of Structural Mechanics, Bauhaus-University

Weimar, 99423 Weimar, Germany continuity is satisfied, meantime the meshless method is easy to form high-order. The approximate function has significant advantages to the numerical solutions for higherorder partial-differential equations.

Rabczuk [16] devised a meshfree method for thin shell analysis for finite strains and arbitrary evolving cracks exploiting the higher order continuity of the EFG shape functions and avoiding any rotational degrees of freedom. Mohammed et al. [17, 18] presented a meshless method to analyze the mechanical response of elastic thin plates. However, since meshless shape functions are commonly rational functions, more integration points are needed to evaluate the weak form. For example, Brebbia [6] employed $6 \times 6$ quadrature points, which significantly reduces the computational efficiency. An interesting alternative to meshless methods is isogeometric analysis (IGA) [19,20], which also fulfills the higher order continuity requirement needed for thin plate analysis. This method takes advantage of NURBS/B-Spline basis functions, which are commonly used in computeraided-design (CAD) to describe the geometries. IGA has been successfully applied to the analysis of plates and shells for instance in [21-25]. As CAD geometries are surface representations, they are particularly suited for plates and shells. 
One difficulty occurs for multi-patch geometries, which are still difficult to deal with.

In this study, we take advantage of nonlocal theories as suggested for instance in nonlocal continuum field theories with various physical fields [26], peridynamics (PD) [27], nonlocal plasticity, (nonlocal) damage mechanics [28] and nonlocal vector calculus [29]. Nonlocal operator method (NOM) $[30,31]$ is a nonlocal numerical method for solving partial differential equations. The method is based on socalled differential operators. In contrast to finite elements, the NOM only needs neighboring points to develop nonlocal derivatives. Similar to the machine learning approach [32, 33], it can solve PDEs directly instead of the need of shape functions, which plays an equivalent role as the derivatives of the shape functions in the meshless methods or the FEM. And, therefore, the complexity of the nonlocal operator method is significantly reduced. The nonlocal strong form can be derived by a variational derivation on the functional defined by nonlocal operators. This paper presents a nonlocal operator method to predict the dynamic response of Kirchhoff plates exploiting the higher order continuity of the NOM.

The paper is organized as follows: We first briefly review the NOM and derive the nonlocal Hessian operator for Kirchhoff plates in Sect. 2. In Sect. 3, we derive the nonlocal dynamic Kirchhoff plate formulation by a variational formulation. Section 4 presents details about the numerical implementation before we demonstrate the performance of the formulation through several benchmark problems in Sect. 5. We conclude the manuscript in Sect. 6.

\section{Outline of nonlocal operator method and derivation of nonlocal Hessian operator for Kirchhoff plate}

\subsection{Outline of nonlocal operator method}

We consider a Kirchhoff plate occupying a domain $\boldsymbol{\Omega}$ as illustrated in Fig. 1. Let us denote the spatial coordinates with $\mathbf{x}_{i}, \boldsymbol{\xi}_{i j}:=\mathbf{x}_{j}-\mathbf{x}_{i}$ is relative position vector from $\mathbf{x}_{i}$ to $\mathbf{x}_{j}$; $w_{i}:=w\left(\mathbf{x}_{i}, t\right)$ and $w_{j}:=w\left(\mathbf{x}_{j}, t\right)$ are the displacement value for $\mathbf{x}_{i}$ and $\mathbf{x}_{j}$, respectively; the relative displacement area for the spatial vector $\boldsymbol{\xi}_{i j}$ is $w_{i j}:=w_{j}-w_{i}$.

The support and the dual-support are two fundamental principles in NOM. The domain, in which every spatial point $\mathbf{x}_{j}$ forms the spatial vector $\boldsymbol{\xi}_{i j}$ from $\mathbf{x}_{i}$ to $\mathbf{x}_{j}$ is called the support $\mathcal{S}_{i}$ of point $\mathbf{x}_{i}$, see Fig. 1b. We can write $\mathcal{S}_{\mathbf{x}}=\left\{\mathbf{x}_{2}, \mathbf{x}_{3}, \mathbf{x}_{5}, \mathbf{x}_{6}\right\}$. The dual-support of $\mathbf{x}_{i}$ is defined as a union of points which supports include $\mathbf{x}$, i.e.

$\mathcal{S}_{i}^{\prime}=\left\{\mathbf{x}_{j} \mid \mathbf{x}_{i} \in \mathcal{S}_{j}\right\}$

The dual-support of point $\mathbf{x}_{j}$ forms the dual-vector $\boldsymbol{\xi}_{i j}^{\prime}\left(=\mathbf{x}_{i}-\mathbf{x}_{j}\right)$ in $\mathcal{S}_{i}^{\prime}$ and is denoted as $\mathcal{S}_{\mathbf{x}}^{\prime}=\left\{\mathbf{x}_{3}, \mathbf{x}_{6}\right\} ; \boldsymbol{\xi}_{i j}^{\prime}$ is the $\mathcal{S}_{j}$ relative position space vector. NOM requires fundamental nonlocal operators that replace the local operators in calculus. Thus, the functional designed to construct a residual and tangent stiffness matrix is formulated in terms of the nonlocal differential operator. The higher order nonlocal operator $\tilde{\partial}_{\alpha} w_{i}$ for the scalar field $w$ in support $\mathcal{S}_{i}$ can be expressed as [34]

Fig. 1 Schematic diagram of NOM

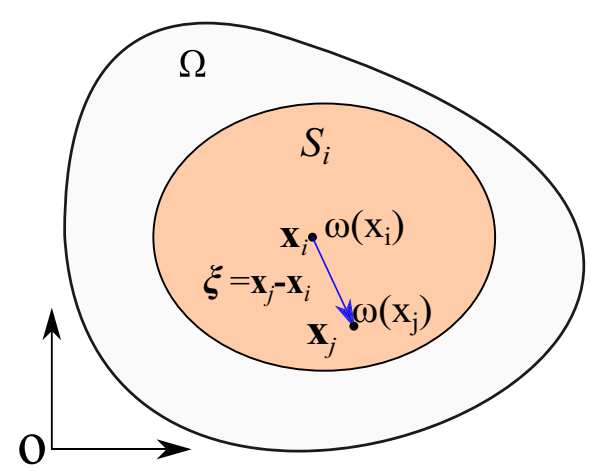

(a)

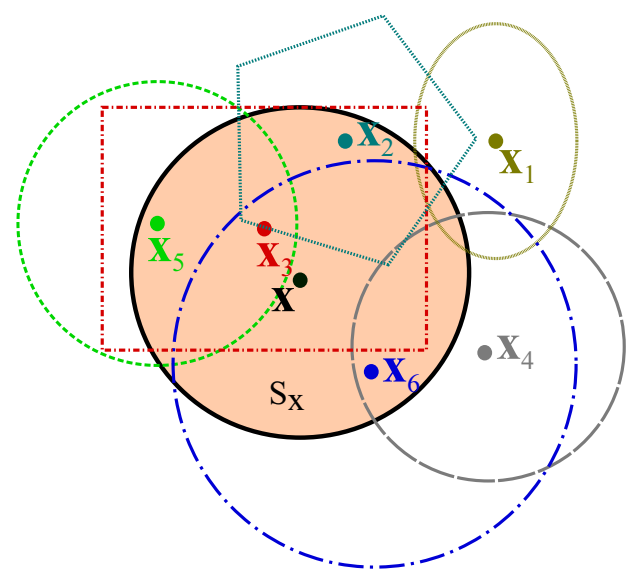

(b) 
$\tilde{\partial}_{\alpha} w_{i}:=\int_{\mathcal{S}_{i}} \phi\left(\boldsymbol{\xi}_{i j}\right) w_{i j} \mathbf{s}_{i j} d V_{j} \cdot\left(\int_{\mathcal{S}_{i}} \phi\left(\boldsymbol{\xi}_{i j}\right) \mathbf{s}_{i j} \mathbf{s}_{i j}^{T} d V_{j}\right)^{-1}$,

where $\phi\left(\boldsymbol{\xi}_{i j}\right)$ represents the weight function, $\boldsymbol{\xi}_{i j}$ represents relative position vector. $\mathbf{s}_{i j}$ represent the list of polynomials. For example, the polynomials and the higher order nonlocal operator in $2 \mathrm{D}$ with maximal second-order derivatives are $\mathbf{s}_{i j}=\left(x_{i j}, y_{i j}, x_{i j}^{2} / 2, x_{i j} y_{i j}, y_{i j}^{2} / 2\right)^{T}$ and $\tilde{\partial}_{\alpha} w_{i}=\left(\frac{\partial w_{i}}{\partial x}, \frac{\partial w_{i}}{\partial y}, \frac{\partial^{2} w_{i}}{\partial x^{2}}, \frac{\partial^{2} w_{i}}{\partial x y}, \frac{\partial^{2} w_{i}}{\partial y^{2}}\right)^{T}$.

Using nodal integration, the higher order nonlocal operator and it's variation for a vector field $w$ in discrete forms are

$\tilde{\partial}_{\alpha} w_{i}=\sum_{j \in \mathcal{S}_{i}} \phi\left(\boldsymbol{\xi}_{i j}\right) w_{i j} \mathbf{s}_{i j} \Delta V_{j} \cdot\left(\sum_{j \in \mathcal{S}_{i}} \phi\left(\boldsymbol{\xi}_{i j}\right) \mathbf{s}_{i j} \mathbf{s}_{i j}^{T} \Delta V_{j}\right)^{-1}$

$\tilde{\partial}_{\alpha} \delta w_{i}=\sum_{j \in \mathcal{S}_{i}} \phi\left(\boldsymbol{\xi}_{i j}\right)\left(\delta w_{j}-\delta w_{i}\right) \boldsymbol{\xi}_{i j} \Delta V_{j} \cdot\left(\sum_{j \in \mathcal{S}_{i}} \phi\left(\boldsymbol{\xi}_{i j}\right) \mathbf{s}_{i j} \mathbf{s}_{i j}^{T} \Delta V_{j}\right)^{-1}$.

We use a penalty energy functional to obtain the linear field of the scalar field to eliminate zero-energy modes. The higher order operator energy functional for a scalar field $w$ at a point $\mathbf{x}_{i}$ is defined as

$\mathcal{F}_{i}^{h g}=\frac{\alpha_{w}}{2 m_{i}} \int_{\mathcal{S}_{i}} \phi\left(\boldsymbol{\xi}_{i j}\right)\left(\mathbf{s}_{i j}^{T} \tilde{\partial}_{\alpha} w_{i}-w_{i j}\right)^{2} d V_{j}$,

where $m_{i}\left(=\int_{\mathcal{S}_{i}} \phi\left(\boldsymbol{\xi}_{i j}\right) \xi_{i j} \otimes \boldsymbol{\xi}_{i j} d V_{j}\right)$ is the normalization coefficient and $\alpha_{w}$ is the penalty coefficient. The nonuniform aspect of the deformation is defined as $\mathbf{s}_{i j}^{T} \tilde{\partial}_{\alpha} w_{i}-w_{i j}$, and the stability of the NOM is greatly enhanced while $\mathbf{s}_{i j}^{T} \tilde{\partial}_{\alpha} w_{i}-w_{i j}$ is enforced explicitly.

\subsection{Derivation of nonlocal Hessian operator for Kirchhoff plate}

In this part, we derive the second-order nonlocal Hessian operator and its variation. The second derivative and its variation in $1 \mathrm{D}$ is given as

$\frac{d^{2} w_{i}}{d x^{2}}=2 \int_{\mathcal{S}_{i}} \phi\left(\xi_{i j}\right) w_{i j}\left(\xi_{i j}^{2}-\frac{K_{3 i}}{K_{2 i}} \xi_{i j}\right) d V_{j} \cdot K_{4 i}^{-1}$

$\tilde{\nabla}^{T} \tilde{\nabla} \delta w_{i}=2 \int_{\mathcal{S}_{i}} \phi\left(\xi_{i j}\right) \delta w_{i j}\left(\xi_{i j}^{2}-\frac{K_{3 i}}{K_{2 i}} \xi_{i j}\right) d V_{j} \cdot K_{4 i}^{-1}$.

To devise the nonlocal Hessian operator in 2D, let us define first the vector $\boldsymbol{\xi}_{i j}=\left(x_{i j}, y_{i j}\right)^{T}$. It can be shown that the second-order shape tensor for point $\mathbf{x}_{i}$ is computed by [30]

$\mathbf{K}_{2 i}=\int_{\mathcal{S}_{i}} \phi\left(\boldsymbol{\xi}_{i j}\right) \boldsymbol{\xi}_{i j} \otimes \boldsymbol{\xi}_{i j} d V_{j}=\int_{\mathcal{S}_{i}} \phi\left(\boldsymbol{\xi}_{i j}\right)\left[\begin{array}{cc}x_{i j}^{2} & x_{i j} y_{i j} \\ x_{i j} y_{i j} & y_{i j}^{2}\end{array}\right] d V_{j}$ and the third-order shape tensor for point $\mathbf{x}_{i}$ by

$$
\begin{aligned}
\mathbf{K}_{3 i}= & \int_{\mathcal{S}_{i}} \phi\left(\boldsymbol{\xi}_{i j}\right) \xi_{i j}^{3} d V_{j} \\
= & \left(\int_{\mathcal{S}_{i}} \phi\left(\boldsymbol{\xi}_{i j}\right) x_{i j}\left[\begin{array}{cc}
x_{i j}^{2} & x_{i j} y_{i j} \\
x_{i j} y_{i j} & y_{i j}^{2}
\end{array}\right] d V_{j},\right. \\
& \left.\int_{\mathcal{S}_{i}} \phi\left(\boldsymbol{\xi}_{i j}\right) y_{i j}\left[\begin{array}{cc}
x_{i j}^{2} & x_{i j} y_{i j} \\
x_{i j} y_{i j} & y_{i j}^{2}
\end{array}\right] d V_{j}\right) \\
= & \left(\int_{\mathcal{S}_{i}} \phi\left(\boldsymbol{\xi}_{i j}\right)\left[\begin{array}{cc}
x_{i j}^{3} & x_{i j}^{2} y_{i j} \\
x_{i j}^{2} y_{i j} & x_{i j} y_{i j}^{2}
\end{array}\right] d V_{j},\right. \\
& \left.\int_{\mathcal{S}_{i}} \phi\left(\boldsymbol{\xi}_{i j}\right)\left[\begin{array}{ll}
x_{i j}^{2} y_{i j} & x_{i j} y_{i j}^{2} \\
x_{i j} y_{i j}^{2} & y_{i j}^{3}
\end{array}\right] d V_{j}\right)=\left(\mathbf{K}_{3 i}^{x}, \mathbf{K}_{3 i}^{y}\right)
\end{aligned}
$$

with $\xi_{i j}^{n}:=\underbrace{\xi_{i j} \otimes \xi_{i j} \otimes \cdots \otimes \xi_{i j}}_{\mathrm{n} \text { terms }}$.

which finally leads to

$\mathbf{K}_{3 i} \mathbf{K}_{2 i}^{-1} \boldsymbol{\xi}_{i j}=\left(\mathbf{K}_{3 i}^{x} \mathbf{K}_{2 i}^{-1} \boldsymbol{\xi}_{i j}, \mathbf{K}_{3 i}^{y} \mathbf{K}_{2 i}^{-1} \boldsymbol{\xi}_{i j}\right)$.

The fourth-order shape tensor for point $\mathbf{x}_{i}$ is computed by

$$
\begin{aligned}
& \mathbf{K}_{4 i}=\int_{\mathcal{S}_{i}} \phi\left(\boldsymbol{\xi}_{i j}\right) \xi_{i j}^{4} d V_{j}
\end{aligned}
$$

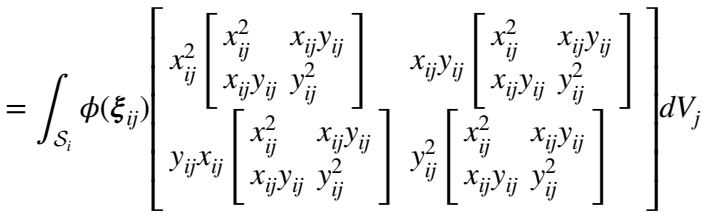

$$
\begin{aligned}
& =\int_{\mathcal{S}_{i}} \phi\left(\boldsymbol{\xi}_{i j}\right)\left[\left[\begin{array}{ll}
x_{i j}^{4} & x_{i j}^{3} y_{i j} \\
x_{i j}^{3} y_{i j} & x_{i j}^{2} y_{i j}^{2} \\
x_{i j}^{3} y_{i j} & x_{i j}^{2} y_{i j}^{2} \\
x_{i j}^{2} y_{i j}^{2} & x_{i j} y_{i j}^{3}
\end{array}\right]\left[\begin{array}{ll}
x_{i j}^{3} y_{i j} & x_{i j}^{2} y_{i j}^{2} \\
x_{i j}^{2} y_{i j}^{2} & x_{i j} y_{i j}^{3} \\
x_{i j}^{2} y_{i j}^{2} & x_{i j} y_{i j}^{3} \\
x_{i j} y_{i j}^{3} & y_{i j}^{4}
\end{array}\right]\right] d V_{j}=\left[\begin{array}{ll}
\mathbf{K}_{4 i}^{x x} & \mathbf{K}_{4 i}^{x y} \\
\mathbf{K}_{4 i}^{y y} & \mathbf{K}_{4 i}^{y y}
\end{array}\right]
\end{aligned}
$$

The second-order Taylor series extension for a scalar field $w$ is given as

$w_{j}=w_{i}+\nabla w_{i} \cdot \xi_{i j}+\frac{1}{2 !} \nabla^{T} \nabla w_{i}: \xi_{i j} \otimes \xi_{i j}+O\left(\left|\xi_{i j}\right|^{3}\right)$

so that we obtain

$w_{i j}=\nabla w_{i} \cdot \xi_{i j}+\frac{1}{2} \nabla^{T} \nabla w_{i}: \xi_{i j} \otimes \xi_{i j}+O\left(\left|\xi_{i j}\right|^{3}\right)$

which finally results in the following Hessian nonlocal operator

$\frac{1}{2} \nabla^{T} \nabla w_{i}: \xi_{i j} \otimes \xi_{i j}=w_{i j}-\nabla w_{i} \cdot \xi_{i j}$

and 


$$
\begin{aligned}
& \frac{1}{2} \nabla^{T} \nabla w_{i}: \int_{\mathcal{S}_{i}} \phi\left(\xi_{i j}\right) \xi_{i j}^{4} d V_{j}=\int_{\mathcal{S}_{i}} \phi\left(\xi_{i j}\right)\left(w_{i j} \xi_{i j} \otimes \xi_{i j}\right. \\
& \left.\quad-\nabla w_{i} \cdot \xi_{i j}^{3}\right) d V_{j} .
\end{aligned}
$$

The weighted tensor $\frac{1}{2} \nabla^{T} \nabla w: \mathbf{K}_{4 i}$ can be simplified to

$$
\begin{aligned}
\frac{1}{2} \nabla^{T} \nabla w_{i}: \mathbf{K}_{4 i}= & \int_{\mathcal{S}_{i}} \phi\left(\boldsymbol{\xi}_{i j}\right)\left(w_{i j} \xi_{i j} \otimes \xi_{i j}-\nabla w_{i} \cdot \xi_{i j}^{3}\right) d V_{j} \\
= & \int_{\mathcal{S}_{i}} \phi\left(\boldsymbol{\xi}_{i j}\right) w_{i j} \boldsymbol{\xi}_{i j} \otimes \xi_{i j} d V_{j} \\
& -\nabla w_{i} \cdot \int_{\mathcal{S}_{i}} \phi\left(\xi_{i j}\right) \xi_{i j}^{3} d V_{j} \\
= & \int_{\mathcal{S}_{i}} \phi\left(\boldsymbol{\xi}_{i j}\right) w_{i j} \xi_{i j} \otimes \xi_{i j} d V_{j} \\
& -\int_{\mathcal{S}_{i}} \phi\left(\boldsymbol{\xi}_{i j}\right) w_{i j} \xi_{i j} d V_{j} \cdot\left(\int_{\mathcal{S}_{i}} \phi\left(\boldsymbol{\xi}_{i j}\right) \boldsymbol{\xi}_{i j} \otimes \xi_{i j} \mathrm{~d} V_{j}\right)^{-1} \mathbf{K}_{3 i} \\
= & \int_{\mathcal{S}_{i}} \phi\left(\boldsymbol{\xi}_{i j}\right) w_{i j} \xi_{i j} \otimes \xi_{i j} d V_{j} \\
& -\int_{\mathcal{S}_{i}} \phi\left(\boldsymbol{\xi}_{i j}\right) w_{i j} \xi_{i j} d V_{j} \cdot \mathbf{K}_{2 i}^{-1} \mathbf{K}_{3 i} \\
= & \int_{\mathcal{S}_{i}} \phi\left(\boldsymbol{\xi}_{i j}\right) w_{i j}\left(\boldsymbol{\xi}_{i j} \otimes \boldsymbol{\xi}_{i j}-\mathbf{K}_{3 i} \mathbf{K}_{2 i}^{-1} \xi_{i j}\right) d V_{j} .
\end{aligned}
$$

Note that the rank of $\mathbf{K}_{4 i}$ is 3 and the 2D nonlocal Hessian operator has only three independent variables where $\frac{\partial^{2} \delta w_{i}}{\partial x \partial y}=\frac{\partial^{2} \delta w_{i}}{\partial y \partial x}$, let $\mathbf{K}_{4 i}^{-1}$ as the pseudo-inverse of $\mathbf{K}_{4 i}$ in this study.

For the scalar field $w$, the 2D nonlocal Hessian operator for point $\mathbf{x}_{i}$ can be written in matrix form as

$\tilde{\nabla}^{T} \tilde{\nabla} w_{i}=\left[\begin{array}{ll}\frac{\partial^{2} w_{i}}{\partial x^{2}} & \frac{\partial^{2} w_{i}}{\partial x \partial y} \\ \frac{\partial^{2} w_{i}}{\partial y \partial x} & \frac{\partial^{2} w_{i}}{\partial y^{2}}\end{array}\right]$

We let $\mathbf{K}_{3 i}^{x} \mathbf{K}_{2 i}^{-1}, \mathbf{K}_{3 i}^{y} \mathbf{K}_{2 i}^{-1}$ and the pseudo-inverse of $\mathbf{K}_{4 i}$ be explicitly written as

$$
\begin{aligned}
& \mathbf{K}_{3 i}^{x} \mathbf{K}_{2 i}^{-1}=\left[\begin{array}{ll}
a_{11} & a_{12} \\
a_{12} & a_{22}
\end{array}\right] ; \mathbf{K}_{3 i}^{y} \mathbf{K}_{2 i}^{-1}=\left[\begin{array}{ll}
b_{11} & b_{12} \\
b_{12} & b_{22}
\end{array}\right] \\
& \left.\mathbf{K}_{4 i}^{-1}=\left[\begin{array}{ll}
c_{1} & c_{2} \\
c_{2} & c_{3}
\end{array}\right]\left[\begin{array}{ll}
c_{2} & c_{3} \\
c_{2} & c_{3} \\
c_{3} & c_{4} \\
c_{3} & c_{4}
\end{array}\right]\left[\begin{array}{ll}
c_{3} & c_{4} \\
c_{4} & c_{5}
\end{array}\right]\right]
\end{aligned}
$$

To facilitate the calculation $\mathbf{K}_{4 i}^{-1}$, we convert $\mathbf{K}_{4 i}$ to a $3 \times 3$ matrix yielding

$$
\left[\begin{array}{lll}
c_{1} & c_{2} & c_{3} \\
c_{2} & c_{3} & c_{4} \\
c_{3} & c_{4} & c_{5}
\end{array}\right]^{-1}=\int_{\mathcal{S}_{i}} \phi\left(\xi_{i j}\right)\left[\begin{array}{ccc}
x_{i j}^{4} & x_{i j}^{3} y_{i j} & x_{i j}^{2} y_{i j}^{2} \\
x_{i j}^{3} y_{i j} & x_{i j}^{2} y_{i j}^{2} & x_{i j} y_{i j}^{3} \\
x_{i j}^{2} y_{i j}^{2} & x_{i j} y_{i j}^{3} & y_{i j}^{4}
\end{array}\right] d V_{j}
$$

Since $\boldsymbol{\xi}_{i j} \otimes \boldsymbol{\xi}_{i j}-\mathbf{K}_{3 i} \mathbf{K}_{2 i}^{-1} \boldsymbol{\xi}_{i j}$ is a matrix $\mathbf{Q}_{2 \times 2}$ with the terms $Q_{12}=Q_{21}$. Remove the repeated terms in matrix $\mathbf{Q}$, and the remain terms $Q_{11}, Q_{12}, Q_{22}$ in matrix $\mathbf{Q}$ can be reconstituted a vector $\mathbf{L}_{3 \times 1}=\left(Q_{11}, Q_{12}, Q_{22}\right)^{T}$. Then Eq.16 can be rewritten as

$$
\begin{gathered}
\int_{\mathcal{S}_{i}} \phi\left(\boldsymbol{\xi}_{i j}\right)\left[\begin{array}{ccc}
x_{i j}^{4} & x_{i j}^{3} y_{i j} & x_{i j}^{2} y_{i j}^{2} \\
x_{i j}^{3} y_{i j} & x_{i j}^{2} y_{i j}^{2} & x_{i j} y_{i j}^{3} \\
x_{i j}^{2} y_{i j}^{2} & x_{i j} y_{i j}^{3} & y_{i j}^{4}
\end{array}\right] d V_{j}\left[\begin{array}{c}
\frac{\partial^{2} w_{i}}{\partial x^{2}} \\
\frac{\partial^{2} w_{i}}{\partial x \partial y} \\
\frac{\partial^{2} w_{i}}{\partial y^{2}}
\end{array}\right] \\
=\int_{\mathcal{S}_{i}} \phi\left(\boldsymbol{\xi}_{i j}\right) w_{i j} \mathbf{L}_{i} d V_{j}
\end{gathered}
$$

Finally, $\left(\boldsymbol{\xi}_{i j} \otimes \boldsymbol{\xi}_{i j}-\mathbf{K}_{3 i} \mathbf{K}_{2 i}^{-1} \boldsymbol{\xi}_{i j}\right): \mathbf{K}_{4 i}^{-1}$ can be obtained by reconstituting terms in Eq.21 and can be expressed as

$$
\left(\boldsymbol{\xi}_{i j} \otimes \boldsymbol{\xi}_{i j}-\mathbf{K}_{3 i} \mathbf{K}_{2 i}^{-1} \boldsymbol{\xi}_{i j}\right): \mathbf{K}_{4 i}^{-1}=\left[\begin{array}{ll}
e_{i 11} & e_{i 12} \\
e_{i 21} & e_{i 22}
\end{array}\right]
$$

where

$$
\begin{aligned}
e_{i 11}= & c_{1}\left(x_{i j}^{2}-a_{11} x_{i j}-a_{12} y_{i j}\right)+c_{2}\left(x_{i j} y_{i j}-a_{12} x_{i j}-a_{22} y_{i j}\right) \\
& +c_{3}\left(y_{i j}^{2}-b_{12} x_{i j}-b_{22} y_{i j}\right) \\
e_{i 12}= & e_{i 21}=c_{2}\left(x_{i j}^{2}-a_{11} x_{i j}-a_{12} y_{i j}\right)+c_{3}\left(-a_{12} x_{i j}-a_{22} y_{i j}\right. \\
& \left.+x_{i j} y_{i j}\right)+c_{4}\left(y_{i j}^{2}-b_{12} x_{i j}-b_{22} y_{i j}\right) \\
e_{i 22}= & c_{3}\left(x_{i j}^{2}-a_{11} x_{i j}-a_{12} y_{i j}\right)+c_{4}\left(-a_{12} x_{i j}-a_{22} y_{i j}\right. \\
& \left.+x_{i j} y_{i j}\right)+c_{5}\left(y_{i j}^{2}-b_{12} x_{i j}-b_{22} y_{i j}\right) .
\end{aligned}
$$

The explicit form of the nonlocal Hessian operator in 2D can finally be expressed by

$\tilde{\nabla}^{T} \tilde{\nabla} w_{i}=2 \int_{\mathcal{S}_{i}} \phi\left(\xi_{i j}\right) w_{i j}\left[\begin{array}{ll}e_{i 11} & e_{i 12} \\ e_{i 21} & e_{i 22}\end{array}\right] d V_{j}$.

Note that $e_{i 11}, e_{i 12}, e_{i 22}$ are calculated for each neighbor in the support domain. As a result, the variation of nonlocal Hessian operator can be obtained in explicit form as

$\tilde{\nabla}^{T} \tilde{\nabla} \delta w_{i}=2 \int_{\mathcal{S}_{i}} \phi\left(\boldsymbol{\xi}_{i j}\right)\left(\delta w_{j}-\delta w_{i}\right)\left[\begin{array}{ll}e_{i 11} & e_{i 12} \\ e_{i 21} & e_{i 22}\end{array}\right] d V_{j}$.

\section{Derivation of nonlocal dynamic Kirchhoff plate formulation}

\subsection{Classical elastic plate theory}

Kirchhoff plate theory assumes that the normal stress in the thickness direction can be ignored and the normal of the midplane of the plate remains normal after deformation. Hence, all stresses and strains can be expressed by the 
deflection $w$ of the midplane of the plate. Considering the plate element shown in Fig. 2, the in-plane displacements $u$ and $v$ can, therefore, be expressed in terms of the first derivatives of $w$, i.e.

$$
\begin{aligned}
u(x, y, z) & =-z \frac{\partial w}{\partial x} ; v(x, y, z) \\
& =-z \frac{\partial w}{\partial y} ; w(x, y, z) \simeq w(x, y, 0) \cong w(x, y) .
\end{aligned}
$$

The strain resultant $\boldsymbol{\kappa}$ can be obtained by

$\boldsymbol{\kappa}=\left[\kappa_{x}, \kappa_{y}, \kappa_{x y}\right]^{T}=\left[-\frac{\partial^{2} w}{\partial x^{2}},-\frac{\partial^{2} w}{\partial y^{2}},-\frac{2 \partial^{2} w}{\partial x \partial y}\right]^{T}$,

where $\kappa_{x}$ and $\kappa_{y}$ indicates the curvature of the midplane of the plate in the x-and $\mathrm{y}$-direction while $\kappa_{x y}$ refers to the torsion, respectively.

The stress resultants of the Kirchhoff plate are given by

$\mathbf{M}=\left[M_{x}, M_{y}, M_{x y}\right]^{T}$,

where $M_{x}$ and $M_{y}$ are the bending moment per unit length around the $\mathrm{y}$ - and negative $\mathrm{x}$-axes, respectively, while $M_{x y}\left(=M_{y x}\right)$ is the torque per unit length.

With a linear stress distribution in the z-direction and assuming a thickness of $t$, the stresses can be computed by

$\sigma_{x}=\frac{12 M_{x}}{t^{3}} z ; \sigma_{y}=\frac{12 M_{y}}{t^{3}} z ; \tau_{x y}=\tau_{y x}=\frac{12 M_{x y}}{t^{3}} z$.

The Cauchy stress tensor can also be expressed in terms of the linear strain tensor assuming Hooke's law:

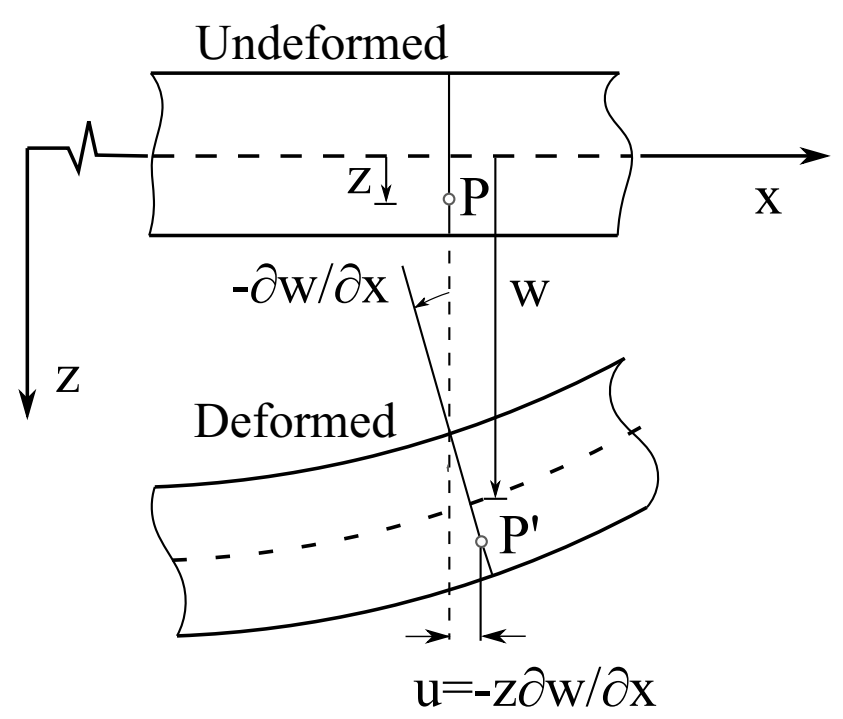

Fig. 2 Deformed configuration of a Kirchhoff plate in bending
$\sigma=\frac{E}{1-v^{2}}\left(v \operatorname{tr} \varepsilon \mathbf{I}_{2 \times 2}+(1-v) \varepsilon\right)$

with

$\varepsilon=\left[\begin{array}{ll}\varepsilon_{11} & \varepsilon_{12} \\ \varepsilon_{21} & \varepsilon_{22}\end{array}\right]$

Finally, the constitutive model can be formulated in terms of the stress and strain resultants by

$\mathbf{M}=\mathbf{D}_{\text {plate }} \boldsymbol{\kappa}$,

where $\mathbf{D}_{\text {plate }}$ is the constitutive matrix defined as

$\mathbf{D}_{\text {plate }}=\frac{E t^{3}}{12\left(1-v^{2}\right)}\left[\begin{array}{ccc}1 & v & 0 \\ v & 1 & 0 \\ 0 & 0 & \frac{1-v}{2}\end{array}\right]=D_{0}\left[\begin{array}{ccc}1 & v & 0 \\ v & 1 & 0 \\ 0 & 0 & \frac{1-v}{2} \\ , & & \end{array}\right]$

where $D_{0}=\frac{E t^{3}}{12\left(1-v^{2}\right)}$ is the Kirchhoff plate's bending stiffness, such that Eq.(30) can be rewritten as

$\overline{\mathbf{M}}=\left[\begin{array}{cc}M_{x} & M_{x y} \\ M_{y x} & M_{y}\end{array}\right]=D_{0}\left(v \operatorname{tr} \overline{\boldsymbol{\kappa}} \boldsymbol{I}_{2 \times 2}+(1-v) \overline{\boldsymbol{\kappa}}\right)$

$\overline{\boldsymbol{\kappa}}=\nabla^{T} \nabla w=\left[\begin{array}{ll}\frac{\partial^{2} w}{\partial x^{2}} & \frac{\partial^{2} w}{\partial x \partial y} \\ \frac{\partial^{2} w}{\partial y \partial x} & \frac{\partial^{2} w}{\partial y^{2}}\end{array}\right]$

\subsection{Nonlocal dynamic Kirchhoff plate formulation}

The total Lagrange energy functional for the Kirchhoff plate can be expressed as

$$
\begin{aligned}
L(\dot{w}, w)= & \int_{\Omega} \frac{1}{2} \rho t \dot{w}^{2} \mathrm{~d} \Omega \\
& -\int_{\Omega}\left(\frac{1}{2} \overline{\mathbf{M}}: \overline{\boldsymbol{\kappa}}-q_{z} w\right) \mathrm{d} \Omega-\int_{\partial \Omega} \overline{M_{n}} \frac{\partial w}{\partial n} d S \\
= & \int_{\Omega} \frac{1}{2} \rho t \dot{w}^{2} \mathrm{~d} \Omega-\int_{\Omega}\left(\frac{1}{2} \overline{\mathbf{M}}: \nabla^{T} \nabla w-q_{z} w\right) \mathrm{d} \Omega \\
& -\int_{\partial \Omega} \overline{M_{n}} \frac{\partial w}{\partial n} d S
\end{aligned}
$$

with $\dot{w}=\frac{\partial w}{\partial t} ; \rho$ is the density of the plate and $q_{z}$ a distributed load in the z-direction. Replacing the local Hessian $\nabla^{T} \nabla w$ with the nonlocal Hessian $\tilde{\nabla}^{T} \tilde{\nabla} w$ in Eq.34, we obtain

$$
\begin{aligned}
L(\dot{w}, w)= & \int_{\Omega} \frac{1}{2} \rho t \dot{w}^{2} \mathrm{~d} \Omega-\int_{\Omega}\left(\frac{1}{2} \overline{\mathbf{M}}: \tilde{\nabla}^{T} \tilde{\nabla} w-q_{z} w\right) \mathrm{d} \Omega \\
& -\int_{\partial \Omega} \overline{M_{n}} \frac{\partial w}{\partial n} d S
\end{aligned}
$$


The integral of the Lagrangian $L$ between two time steps $t_{1}$ and $t_{2}$ is $F=\int_{t_{1}}^{t_{2}} L(\dot{w}, w) d t$. According to the principle of least action, we can write

$$
\begin{aligned}
& F=\int_{t_{1}}^{t_{2}} \int_{\Omega} \frac{1}{2} \rho t \dot{w}^{2} \mathrm{~d} \Omega \mathrm{d} t-\int_{t_{1}}^{t_{2}} \int_{\Omega}\left(\frac{1}{2} \overline{\mathbf{M}}: \tilde{\nabla}^{T} \tilde{\nabla} w-q_{z} w\right) d \Omega \mathrm{d} t \\
&-\int_{t_{1}}^{t_{2}} \int_{\partial \Omega} \overline{M_{n}} \frac{\partial w}{\partial n} d S \mathrm{~d} t .
\end{aligned}
$$

Omitting the external work term $\int_{t_{1}}^{t_{2}} \int_{\partial \Omega} \overline{M_{n}} \frac{\partial w}{\partial n} d S \mathrm{~d} t$, the first variation of $\delta_{F}$ leads to

$$
\begin{aligned}
\delta_{F}= & \int_{t_{1}}^{t_{2}} \int_{\Omega}\left(\rho t \dot{w} \cdot \delta \dot{w}-\overline{\mathbf{M}}: \tilde{\nabla}^{T} \tilde{\nabla} \delta w+q_{z} \delta w\right) \mathrm{d} \Omega \mathrm{d} t \\
= & \int_{t_{1}}^{t_{2}} \int_{\Omega}\left(-\rho_{i} t \ddot{w}_{i} \cdot \delta w_{i}-\overline{\mathbf{M}_{i}}: \tilde{\nabla}^{T} \tilde{\nabla} \delta w_{i}+q_{z} \delta w_{i}\right) \mathrm{d} \Omega \mathrm{d} t \\
= & \int_{t_{1}}^{t_{2}} \int_{\Omega}\left(-\rho_{i} t \ddot{w}_{i} \cdot \delta w_{i}-\overline{\mathbf{M}_{i}}:\left[2 \int _ { \mathcal { S } _ { i } } \phi ( \boldsymbol { \xi } _ { i j } ) \left(\delta w _ { i j } \left(\boldsymbol{\xi}_{i j} \otimes \boldsymbol{\xi}_{i j}\right.\right.\right.\right. \\
& \left.\left.\left.-\mathbf{K}_{3 i} \mathbf{K}_{2 i}^{-1} \boldsymbol{\xi}_{i j}\right) d V_{j}: \mathbf{K}_{4 i}^{-1}\right]+q_{z} \delta w_{i}\right) \mathrm{d} \Omega \mathrm{d} t \\
= & \int_{t_{1}}^{t_{2}} \int_{\Omega}\left(-\rho_{i} t \ddot{w}_{i} \cdot \delta w_{i}-2 \int_{\mathcal{S}_{i}} \overline{\mathbf{M}}_{i}\right. \\
& \left.\quad \phi\left(\boldsymbol{\xi}_{i j}\right)\left(\delta w_{j}-\delta w_{i}\right)\left(\boldsymbol{\xi}_{i j} \otimes \boldsymbol{\xi}_{i j}-\mathbf{K}_{3 i} \mathbf{K}_{2 i}^{-1} \boldsymbol{\xi}_{i j}\right) d V_{j}: \mathbf{K}_{4 i}^{-1}+q_{z} \delta w_{i}\right) \mathrm{d} \Omega \mathrm{d} t \\
= & \int_{t_{1}}^{t_{2}} \int_{\Omega}\left(-\rho_{i} t \ddot{w}_{i} \cdot \delta w_{i}-2\left\{\int _ { \mathcal { S } _ { i } ^ { \prime } } \left[\overline{\mathbf{M}}_{j} \phi\left(\boldsymbol{\xi}_{j i}\right) \delta w_{i}\right.\right.\right. \\
& \left.\left(\boldsymbol{\xi}_{j i} \otimes \boldsymbol{\xi}_{j i}-\mathbf{K}_{3 i} \mathbf{K}_{2 i}^{-1} \boldsymbol{\xi}_{j i}\right): \mathbf{K}_{4 i}^{-1}\right] d V_{j}- \\
& \left.\left.\int_{\mathcal{S}_{i}}\left[\overline{\mathbf{M}_{i}} \phi\left(\boldsymbol{\xi}_{i j}\right) \delta w_{i}\left(\boldsymbol{\xi}_{i j} \otimes \boldsymbol{\xi}_{i j}-\mathbf{K}_{3 j} \mathbf{K}_{2 j}^{-1} \boldsymbol{\xi}_{i j}\right): \mathbf{K}_{4 j}^{-1}\right] d V_{j}\right\}+q_{z} \delta w_{i}\right) \mathrm{d} \Omega \mathrm{d} t
\end{aligned}
$$

According to Eq.23, we devise the explicit form of $\tilde{\nabla}^{T} \tilde{\nabla}: \overline{\mathbf{M}}_{i}$

$$
\begin{gathered}
\tilde{\nabla}^{T} \tilde{\nabla}: \overline{\mathbf{M}_{i}}=2 \int_{\mathcal{S}_{i}} \phi\left(\boldsymbol{\xi}_{i j}\right) \overline{\mathbf{M}_{i}} \cdot\left[\begin{array}{ll}
e_{i 11} & e_{i 12} \\
e_{i 21} & e_{i 22}
\end{array}\right] d V_{j} \\
-2 \int_{\mathcal{S}_{i}^{\prime}} \phi\left(\boldsymbol{\xi}_{j i}\right) \overline{\mathbf{M}_{j}} \cdot\left[\begin{array}{ll}
e_{j 11} & e_{j 12} \\
e_{j 21} & e_{j 22}
\end{array}\right] d V_{j} .
\end{gathered}
$$

As Eq. 37 suffers from zero-energy modes, we introduce the so-called nonlocal operator energy functional, which is described in the next section. where the boundary condition $\delta w\left(t_{1}\right)=0, \delta w\left(t_{2}\right)=0$ is considered in the above derivation. According to Hamilton's principle, for any $\delta w_{i}$, the first variation of the functional $F$ should be zero, which leads to

$$
\begin{aligned}
& 2 \int_{\mathcal{S}_{i}} \overline{\mathbf{M}_{i}} \phi\left(\boldsymbol{\xi}_{i j}\right)\left(\boldsymbol{\xi}_{i j} \otimes \boldsymbol{\xi}_{i j}-\mathbf{K}_{3 i} \mathbf{K}_{2 i}^{-1} \boldsymbol{\xi}_{i j}\right): \mathbf{K}_{4 i}^{-1} d V_{j} \\
& \quad-2 \int_{\mathcal{S}_{i}^{\prime}} \overline{\mathbf{M}_{j}} \phi\left(\boldsymbol{\xi}_{j i}\right)\left(\boldsymbol{\xi}_{j i} \otimes \boldsymbol{\xi}_{j i}-\mathbf{K}_{3 j} \mathbf{K}_{2 j}^{-1} \boldsymbol{\xi}_{j i}\right): \mathbf{K}_{4 j}^{-1} d V_{j} \\
& \quad+q_{z}=\rho_{i} t \ddot{w}_{i} \quad \forall \mathbf{x}_{i} \in \Omega .
\end{aligned}
$$

The nonlocal form is correlated to the local form by

\subsection{Operator energy functional}

For the Kirchhoff plate, the maximal order of partial derivatives in Eq.37 is two, hence we select the second order of nonlocal operators in Eq.5. The operator energy functional for second order nonlocal operators of a scalar field $w$ for point $\mathbf{x}_{i}$ can be expressed as

$\mathcal{F}_{i}^{h g}=\frac{\alpha_{w}}{2 m_{i}} \int_{\mathcal{S}_{i}} \phi\left(\boldsymbol{\xi}_{i j}\right)\left(\mathbf{s}_{i j}^{T} \tilde{\partial}_{\alpha} w_{i}-w_{i j}\right)^{2} d V_{j}$,

$$
\begin{aligned}
& \tilde{\nabla}^{T} \tilde{\nabla}: \overline{\mathbf{M}_{i}} \underset{\text { Nonlocal } \rightarrow \text { Local }}{\stackrel{\text { Local } \rightarrow \text { Nonlocal }}{\rightleftharpoons}} 2 \int_{\mathcal{S}_{i}} \overline{\mathbf{M}_{i}} \phi\left(\boldsymbol{\xi}_{i j}\right)\left(\boldsymbol{\xi}_{i j} \otimes \boldsymbol{\xi}_{i j}-\mathbf{K}_{3 i} \mathbf{K}_{2 i}^{-1} \boldsymbol{\xi}_{i j}\right): \mathbf{K}_{4 i}^{-1} d V_{j}- \\
& 2 \int_{\mathcal{S}_{i}^{\prime}} \overline{\mathbf{M}_{j}} \phi\left(\boldsymbol{\xi}_{j i}\right)\left(\boldsymbol{\xi}_{j i} \otimes \boldsymbol{\xi}_{j i}-\mathbf{K}_{3 j} \mathbf{K}_{2 j}^{-1} \boldsymbol{\xi}_{j i}\right): \mathbf{K}_{4 j}^{-1} d V_{j}
\end{aligned}
$$


where

$\mathbf{s}_{i j}=\left(x_{i j}, y_{i j}, x_{i j}^{2} / 2, x_{i j} y_{i j}, y_{i j}^{2} / 2\right)^{T}$,

$\tilde{\partial}_{\alpha} w_{i}=\left(\frac{\partial w_{i}}{\partial x}, \frac{\partial w_{i}}{\partial y}, \frac{\partial^{2} w_{i}}{\partial x^{2}}, \frac{\partial^{2} w_{i}}{\partial x y}, \frac{\partial^{2} w_{i}}{\partial y^{2}}\right)^{T}$.

The first variation of $\mathcal{F}_{i}^{h g}$ is

$$
\begin{aligned}
\delta \mathcal{F}_{i}^{h g}= & \frac{\alpha_{w}}{m_{i}} \int_{\mathcal{S}_{i}} \phi\left(\boldsymbol{\xi}_{i j}\right)\left(\mathbf{s}_{i j}^{T} \tilde{\partial}_{\alpha} w_{i}-w_{i j}\right)^{T}\left(\tilde{\partial}_{\alpha} \delta w_{i} \mathbf{s}_{i j}^{T}-\delta w_{i j}\right) d V_{j} \\
= & \frac{\alpha_{w}}{m_{i}} \int_{\mathcal{S}_{i}} \phi\left(\boldsymbol{\xi}_{i j}\right)\left(\mathbf{s}_{i j}^{T} \tilde{\partial}_{\alpha} w_{i}-w_{i j}\right)^{T} \tilde{\partial}_{\alpha} \delta w_{i} \mathbf{s}_{i j}^{T} d V_{j} \\
& -\frac{\alpha_{w}}{m_{i}} \int_{\mathcal{S}_{i}} \phi\left(\boldsymbol{\xi}_{i j}\right)\left(\mathbf{s}_{i j}^{T} \tilde{\partial}_{\alpha} w_{i}-w_{i j}\right)^{T} \delta w_{i j} d V_{j} \\
= & \frac{\alpha_{w}}{m_{i}} \int_{\mathcal{S}_{i}} \phi\left(\boldsymbol{\xi}_{i j}\right)\left(\mathbf{s}_{i j}^{T} \tilde{\partial}_{\alpha} w_{i}-w_{i j}\right) \mathbf{s}_{i j}^{T} d V_{j} \cdot \tilde{\partial}_{\alpha} \delta w_{i} \\
& -\frac{\alpha_{w}}{m_{i}} \int_{\mathcal{S}_{i}} \phi\left(\boldsymbol{\xi}_{i j}\right)\left(\mathbf{s}_{i j}^{T} \tilde{\partial}_{\alpha} w_{i}-w_{i j}\right)^{T} \delta w_{i j} d V_{j} \\
= & \frac{\alpha_{w}}{m_{i}}\left(\tilde{\partial}_{\alpha} w_{i} \int_{\mathcal{S}_{i}} \phi\left(\boldsymbol{\xi}_{i j}\right) \mathbf{s}_{i j} \mathbf{s}_{i j}^{T} d V_{j}-\int_{\mathcal{S}_{i}} \phi\left(\boldsymbol{\xi}_{i j}\right) w_{i j} \mathbf{s}_{i j}^{T} d V_{j}\right) \cdot \tilde{\partial}_{\alpha} \delta w_{i}- \\
& \frac{\alpha_{w}}{m_{i}} \int_{\mathcal{S}_{i}} \phi\left(\boldsymbol{\xi}_{i j}\right)\left(\mathbf{s}_{i j}^{T} \tilde{\partial}_{\alpha} w_{i}-w_{i j}\right)^{T} \delta w_{i j} d V_{j} \\
= & \frac{\alpha_{w}}{m_{i}}\left(\int_{\mathcal{S}_{i}} \phi\left(\boldsymbol{\xi}_{i j}\right) w_{i j} \mathbf{s}_{i j}^{T} d V_{j}-\int_{\mathcal{S}_{i}} \phi\left(\boldsymbol{\xi}_{i j}\right) w_{i j} \mathbf{s}_{i j}^{T} d V_{j}\right) \cdot \tilde{\partial}_{\alpha} \delta w_{i}- \\
& \frac{\alpha_{w}}{m_{i}} \int_{\mathcal{S}_{i}} \phi\left(\boldsymbol{\xi}_{i j}\right)\left(\mathbf{s}_{i j}^{T} \tilde{\partial}_{\alpha} w_{i}-w_{i j}\right)^{T} \delta w_{i j} d V_{j} \\
= & \frac{\alpha_{w}}{m_{i}} \int_{\mathcal{S}_{i}} \phi\left(\boldsymbol{\xi}_{i j}\right)\left(w_{i j}-\mathbf{s}_{i j}^{T} \tilde{\partial}_{\alpha} w_{i}\right)^{T}\left(\delta w_{j}-\delta w_{i}\right) d V_{j} .
\end{aligned}
$$

Taking the variation of $\int_{\Omega} \mathcal{F}_{i}^{h g} d V_{i}$ yields

$$
\begin{aligned}
& \int_{\Omega} \mathcal{F}_{i}^{h g} d V_{i}=\int_{\Omega} \frac{\alpha_{w}}{m_{i}} \int_{\mathcal{S}_{i}} \phi\left(\boldsymbol{\xi}_{i j}\right)\left(w_{i j}-\mathbf{s}_{i j}^{T} \tilde{\partial}_{\alpha} w_{i}\right)^{T} \\
& \quad\left(\delta w_{j}-\delta w_{i}\right) d V_{j} d V_{i} \\
& =\int_{\Omega}\left(\int_{\mathcal{S}_{i}^{\prime}} \frac{\alpha_{w}}{m_{j}} \phi\left(\boldsymbol{\xi}_{j i}\right)\left(w_{j i}-\tilde{\partial}_{\alpha} w_{j} \mathbf{s}_{j i}^{T}\right) d V_{j}\right. \\
& \left.-\int_{\mathcal{S}_{i}} \frac{\alpha_{w}}{m_{i}} \phi\left(\boldsymbol{\xi}_{i j}\right)\left(w_{i j}-\tilde{\partial}_{\alpha} w_{i} \mathbf{s}_{i j}^{T}\right) d V_{j}\right) \delta w_{i} d V_{i} .
\end{aligned}
$$

For the scalar field $w$, the internal force due to the operator energy functional is given by

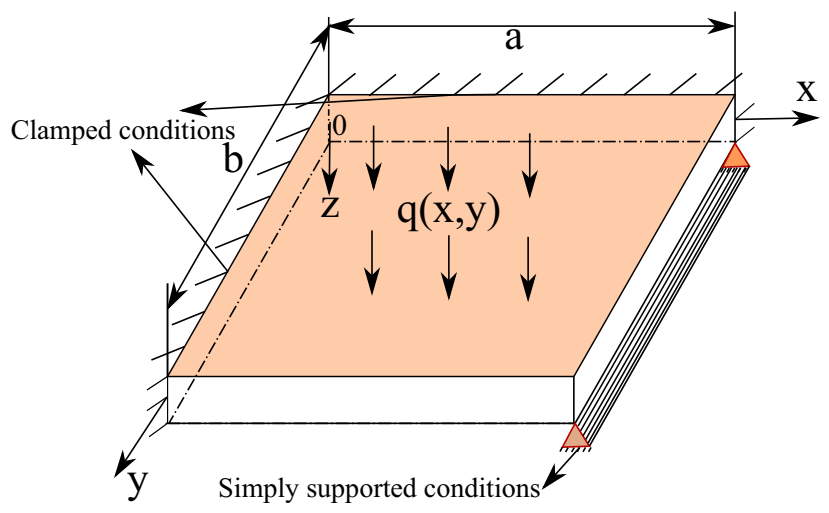

Fig. 3 The Kirchhoff plate bending problem's boundary conditions

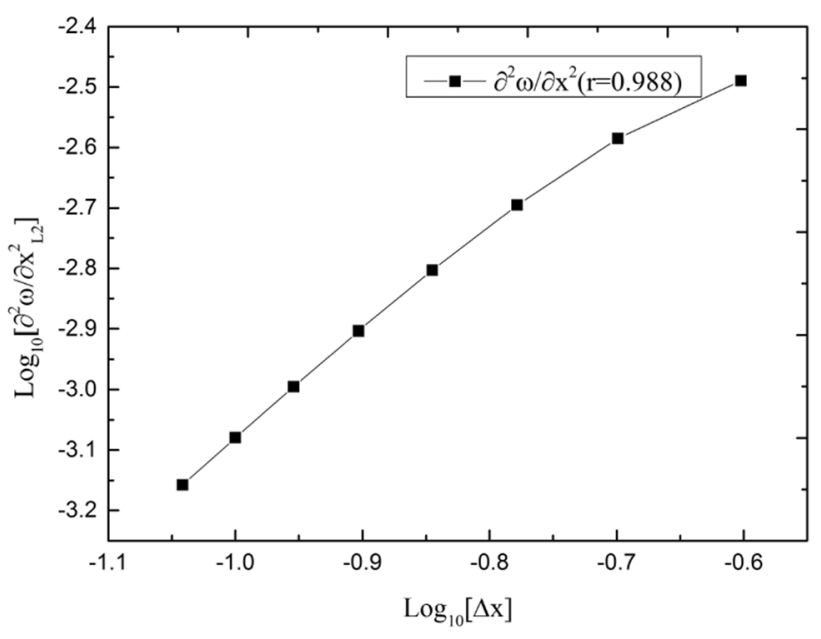

Fig. 4 The L2-norm's convergence for $\frac{\partial^{2} w}{\partial x^{2}}$

$\int_{\mathcal{S}_{i}^{\prime}} \boldsymbol{f}_{j i} d V_{j}-\int_{\mathcal{S}_{i}} \boldsymbol{f}_{i j} d V_{j}$

where $\boldsymbol{f}_{i j}=\frac{\alpha_{w}}{m_{i}} \phi\left(\boldsymbol{\xi}_{i j}\right)\left(w_{i j}-\tilde{\partial}_{\alpha} w_{i} \mathbf{s}_{i j}^{T}\right)$ indicates the zero-energy internal force. Finally, the correspondence between local and nonlocal formulation and the operator functional enhanced governing equation of Kirchhoff plate can be expressed as 

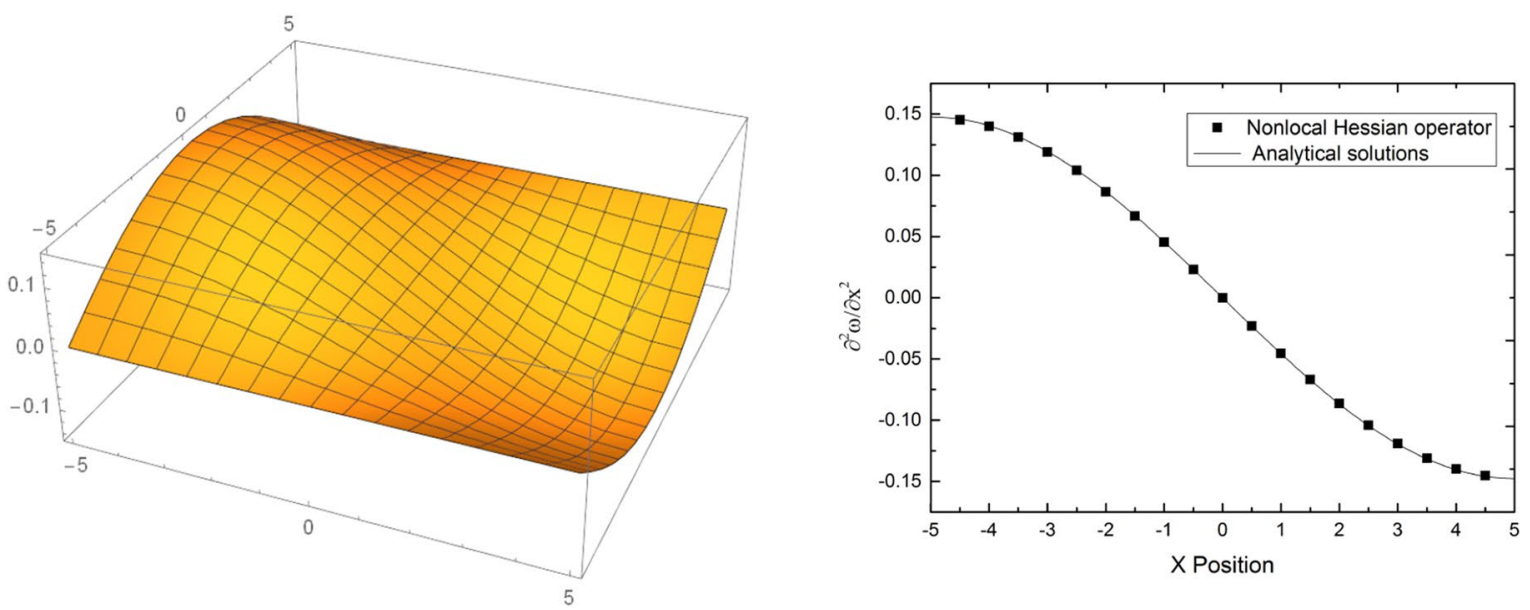

(a)
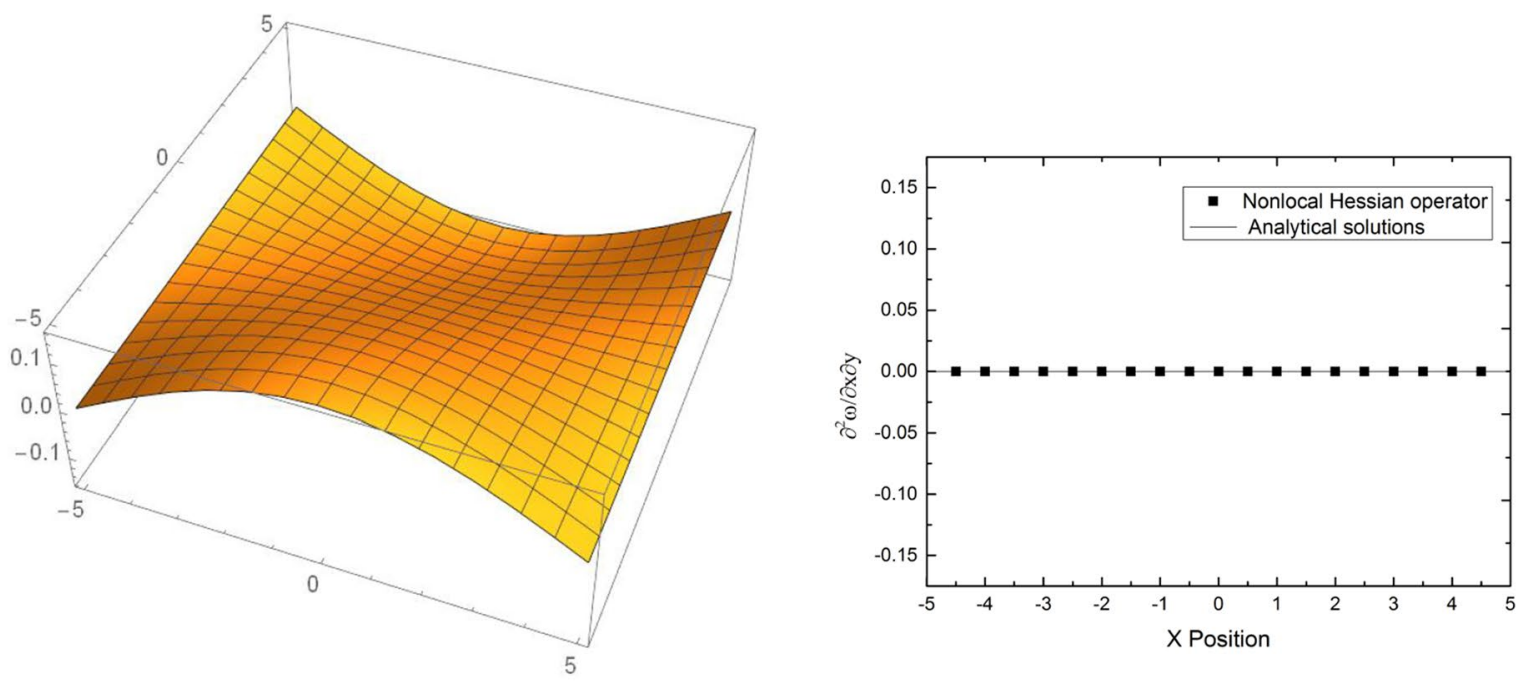

(b)
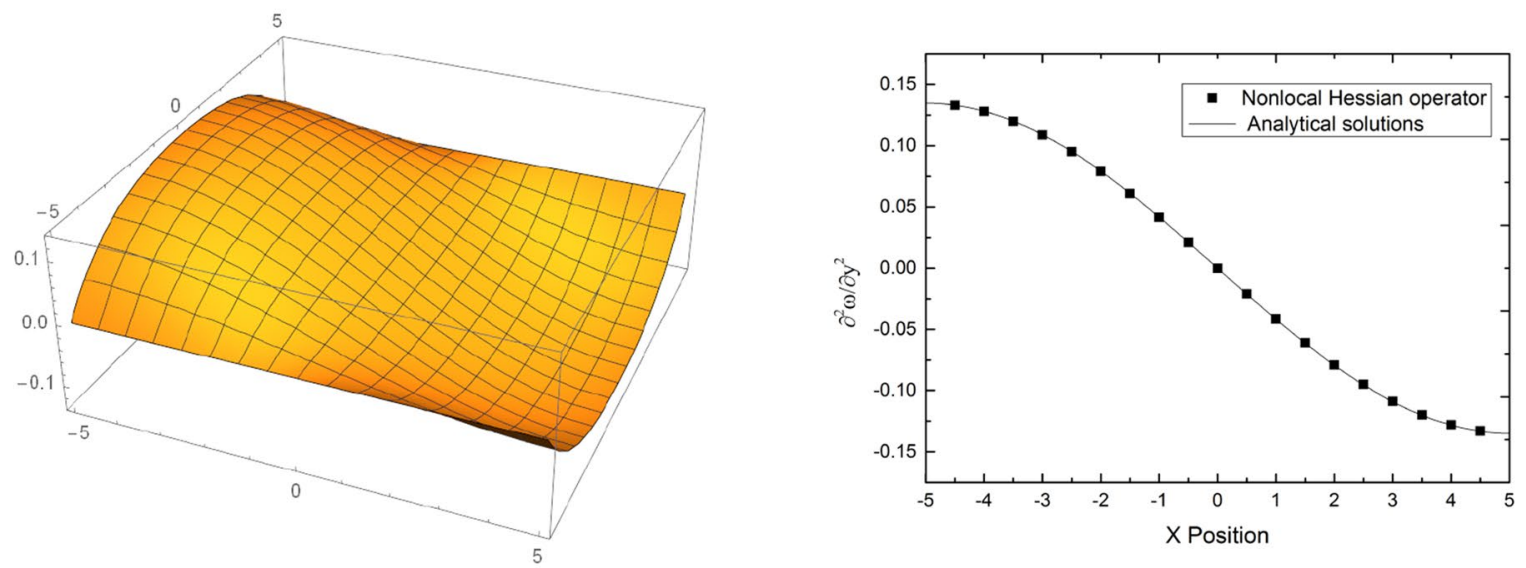

(c) 
4 Fig. 5 Deflection curve of analytical solutions and relative error $(\mathrm{y}=0)$. a Contour of the deflection $\frac{\partial^{2} w}{\partial x^{2}}$ for $\Upsilon=1 ; \mathbf{b}$ Contour of the deflection $\frac{\partial^{2} w}{\partial x \partial y}$ for $\Upsilon=1$; c Contour of the deflection $\frac{\partial^{2} w}{\partial y^{2}}$ for $\Upsilon=1$ $\left.w\right|_{x=a}=0 ;\left.M_{x}\right|_{x=a}=-\left.D_{0} \frac{\partial^{2} w}{\partial x^{2}}\right|_{x=a}=0$

Similarly, parallel to the $x$ - axis (as $y=b$ simply supported boundary), the boundary conditions is

$$
\begin{aligned}
& \tilde{\nabla}^{T} \tilde{\nabla}: \overline{\mathbf{M}_{i}} \underset{\text { Nonlocal } \rightarrow \text { Local }}{\stackrel{\text { Local } \rightarrow \text { Nonlocal }}{\rightleftharpoons}} \int_{\mathcal{S}_{i}}\left(2 \phi\left(\boldsymbol{\xi}_{i j}\right) \overline{\mathbf{M}_{i}} \cdot\left[\begin{array}{ll}
e_{i 11} & e_{i 12} \\
e_{i 21} & e_{i 22}
\end{array}\right]+\boldsymbol{f}_{i j}\right) d V_{j}- \\
& \int_{\mathcal{S}_{i}^{\prime}}\left(2 \phi\left(\boldsymbol{\xi}_{j i}\right) \overline{\mathbf{M}_{j}} \cdot\left[\begin{array}{ll}
e_{j 11} & e_{j 12} \\
e_{j 21} & e_{j 22}
\end{array}\right]+\boldsymbol{f}_{j i}\right) d V_{j}
\end{aligned}
$$

$$
\begin{aligned}
& \int_{\mathcal{S}_{i}}\left(2 \phi\left(\boldsymbol{\xi}_{i j}\right) \overline{\mathbf{M}_{i}} \cdot\left[\begin{array}{ll}
e_{i 11} & e_{i 12} \\
e_{i 21} & e_{i 22}
\end{array}\right]+\frac{\alpha_{w}}{m_{i}} \phi\left(\boldsymbol{\xi}_{i j}\right)\left(w_{i j}-\tilde{\partial}_{\alpha} w_{i} \mathbf{s}_{i j}^{T}\right)\right) d V_{j}- \\
& \int_{\mathcal{S}_{i}^{\prime}}\left(2 \phi\left(\boldsymbol{\xi}_{j i}\right) \overline{\mathbf{M}_{j}} \cdot\left[\begin{array}{ll}
e_{j 11} & e_{j 12} \\
e_{j 21} & e_{j 22}
\end{array}\right]+\frac{\alpha_{w}}{m_{j}} \phi\left(\boldsymbol{\xi}_{j i}\right)\left(w_{j i}-\tilde{\partial}_{\alpha} w_{j} \mathbf{s}_{j i}^{T}\right)\right) \\
& d V_{j}+q_{z}=\rho_{i} t \ddot{w}_{i} \quad \forall \mathbf{x}_{i} \in \Omega .
\end{aligned}
$$

\subsection{Kirchhoff plate boundary conditions}

Let us consider the boundary conditions shown in Fig. 3, which can be classified into:

1. Clamped boundary conditions, where the deflection and the slope of the mid-plane is zero. The positioning shift $\bar{w}$ and the section rotation $\bar{\theta}$ are both zero. The boundary conditions are in the direction parallel to the $y$-axis (as $x=0$ clamped boundary):

$\left.w\right|_{x=0}=0 ;\left.\frac{\partial w}{\partial x}\right|_{x=0}=0$.

Parallel to the $x$-axis (as $y=0$ clamped boundary), the Kirchhoff plate boundary conditions is

$\left.w\right|_{y=0}=0 ;\left.\frac{\partial w}{\partial y}\right|_{y=0}=0$.

2. Simply supported Kirchhoff plate boundary conditions where the plate is free to rotate about a line but prevented from deflecting. The positioning shift $\bar{w}$ and moment $\overline{M_{n}}$ value is zero: parallel to the $y$-axis (as $x=a$ simply supported boundary), the boundary conditions is

$\left.w\right|_{x=a}=0 ;\left.M_{x}\right|_{x=a}=-D_{0}\left(\frac{\partial^{2} w}{\partial x^{2}}+v \frac{\partial^{2} w}{\partial y^{2}}\right)_{x=a}=0$

and Eq.46 can be written as

$$
\begin{aligned}
& \left.w\right|_{y=b} \quad=0 ;\left.M_{y}\right|_{y=b}=-D_{0}\left(\nu \frac{\partial^{2} w}{\partial x^{2}}+\frac{\partial^{2} w}{\partial y^{2}}\right)_{y=b} \\
& =-\left.D_{0} \frac{\partial^{2} w}{\partial y^{2}}\right|_{y=b}=0 .
\end{aligned}
$$

\section{Numerical implementation}

The domain $\Omega$ is decomposed into $N$ points occupying a volume $\Delta V_{i}$ :

$\Omega=\sum_{i=1}^{N} \Delta V_{i}$

For each point, the support is denoted by

$\mathcal{S}_{i}=\left\{i, j_{1}, j_{2}, \ldots, j_{n_{i}}\right\}$,

where $j_{1}, \ldots, j_{k}, \ldots, j_{n_{i}}$ are the global indices of the neighbors of point $\mathbf{x}_{i}$ and $n_{i}$ represents the number of neighbors in support domain $\mathcal{S}_{i}$. The out-of-plane transversal force between points are computed by

$$
\begin{aligned}
\boldsymbol{T}_{i j}= & \left(2 \overline{\mathbf{M}_{i}} \phi\left(\boldsymbol{\xi}_{i j}\right)\left(\boldsymbol{\xi}_{i j} \otimes \boldsymbol{\xi}_{i j}-\mathbf{K}_{3 i} \mathbf{K}_{2 i}^{-1} \boldsymbol{\xi}_{i j}\right): \mathbf{K}_{4 i}^{-1}+\boldsymbol{f}_{i j}\right) \\
& \Delta V_{i} \Delta V_{j} \\
= & \left(2 \phi\left(\boldsymbol{\xi}_{i j}\right) \overline{\mathbf{M}_{i}} \cdot\left[\begin{array}{ll}
e_{i 11} & e_{i 12} \\
e_{i 21} & e_{i 22}
\end{array}\right]+\frac{\alpha_{w}}{m_{i}} \phi\left(\boldsymbol{\xi}_{i j}\right)\left(w_{i j}-\tilde{\partial}_{\alpha} w_{i} \mathbf{s}_{i j}^{T}\right)\right) \\
& \Delta V_{i} \Delta V_{j} \\
\boldsymbol{T}_{j i}= & \left(2 \overline{\mathbf{M}_{j}} \phi\left(\boldsymbol{\xi}_{j i}\right)\left(\boldsymbol{\xi}_{j i} \otimes \boldsymbol{\xi}_{j i}-\mathbf{K}_{3 j} \mathbf{K}_{2 j}^{-1} \boldsymbol{\xi}_{j i}\right): \mathbf{K}_{4 j}^{-1}+\boldsymbol{f}_{j i}\right) \Delta V_{i} \Delta V_{j} \\
= & \left(2 \phi\left(\boldsymbol{\xi}_{j i} \overline{\mathbf{M}_{j}} \cdot\left[\begin{array}{ll}
e_{j 11} & e_{j 12} \\
e_{j 21} & e_{j 22}
\end{array}\right]+\frac{\alpha_{w}}{m_{j}} \phi\left(\boldsymbol{\xi}_{j i}\right)\left(w_{j i}-\tilde{\partial}_{\alpha} w_{j} \mathbf{s}_{j i}^{T}\right)\right)\right. \\
& \Delta V_{i} \Delta V_{j} .
\end{aligned}
$$



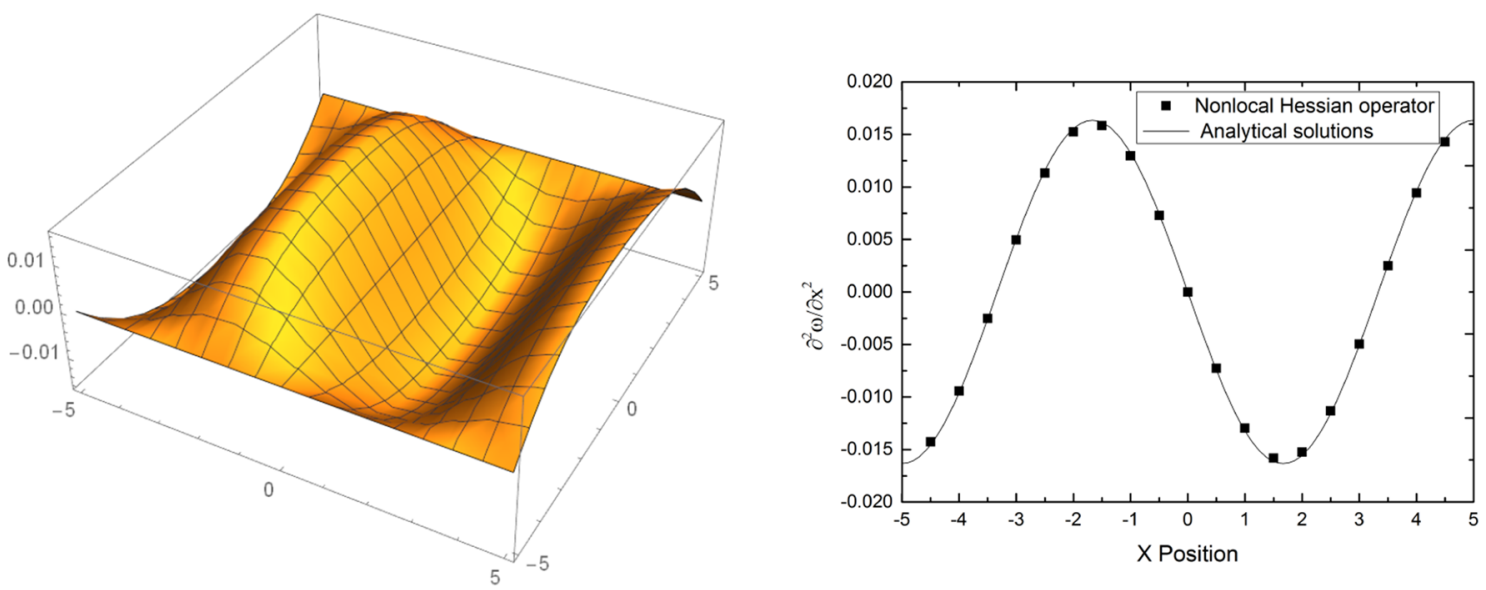

(a)
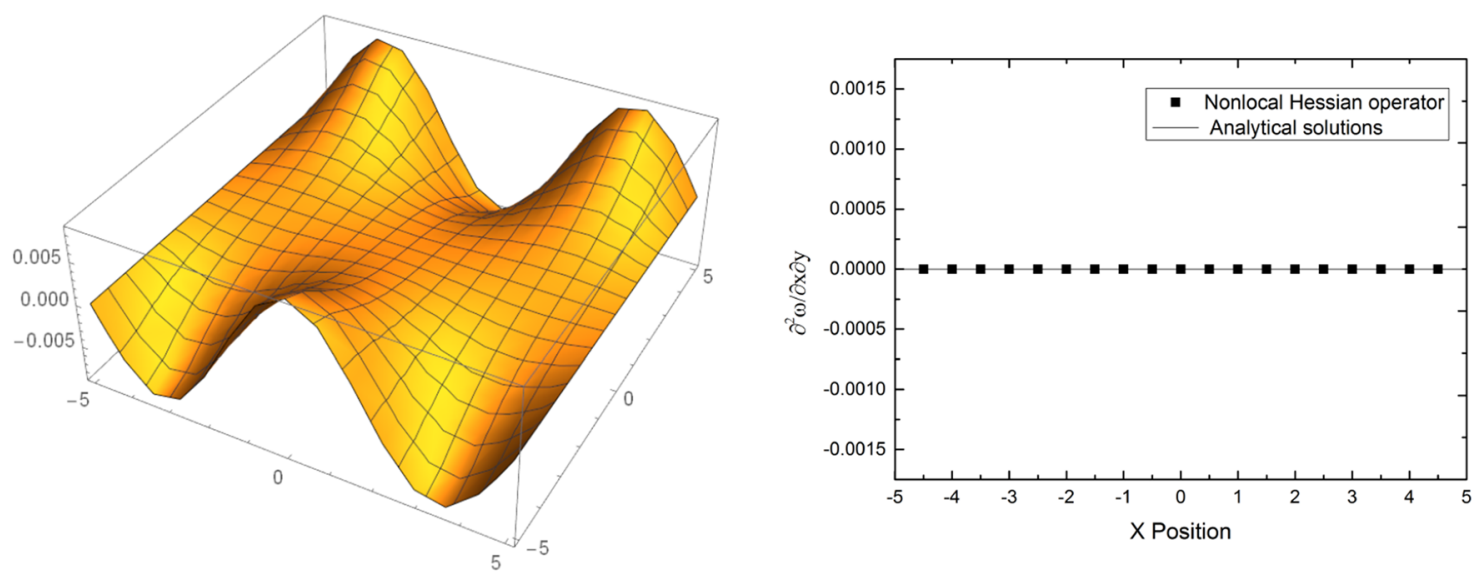

(b)
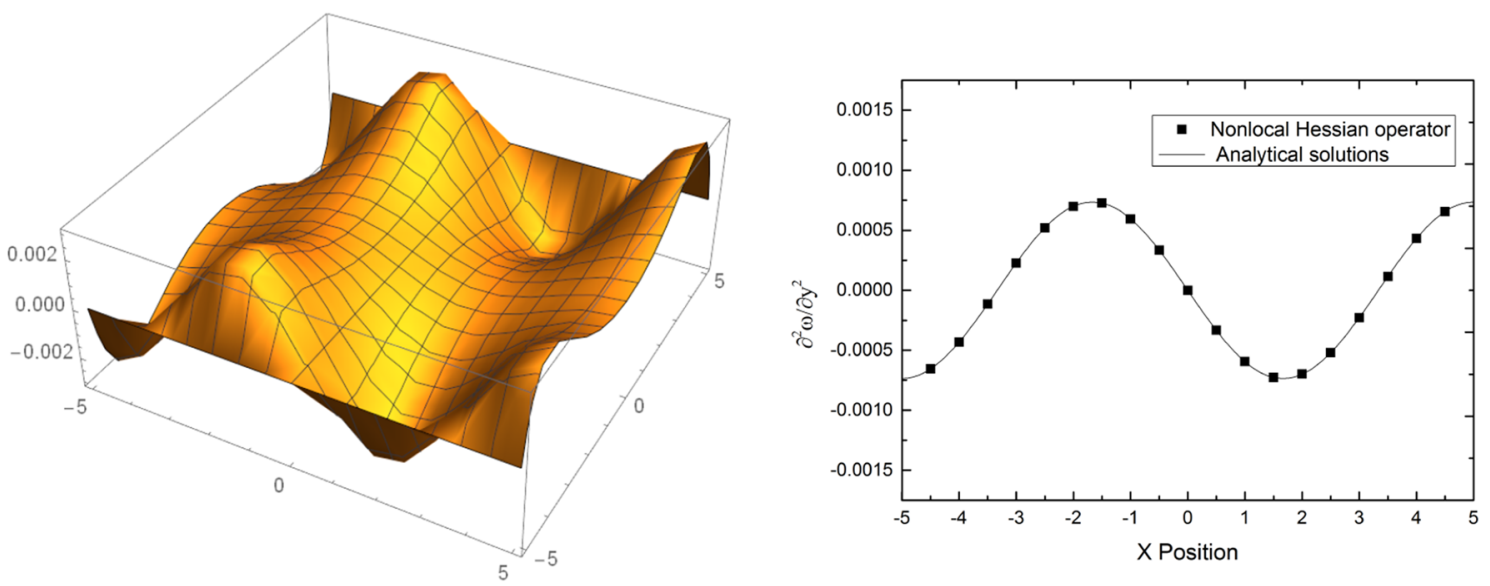

(c)

Fig. 6 Deflection curve of analytical solutions and relative error $(\mathrm{y}=0)$. a Contour of the deflection $\frac{\partial^{2} w}{\partial x^{2}}$ for $\Upsilon=3$; $\mathbf{b}$ Contour of the deflection $\frac{\partial^{2} w}{\partial x \partial y}$ for $\Upsilon=3$; $\mathbf{c}$ Contour of the deflection $\frac{\partial^{2} w}{\partial y^{2}}$ for $\Upsilon=3$ 
Finally, we get the internal force $\mathcal{P}_{i j}$ between points as

$\mathcal{P}_{i j}=\sum_{j \in \mathcal{S}_{i}} \boldsymbol{T}_{i j}-\sum_{j \in \mathcal{S}_{i}^{\prime}} \boldsymbol{T}_{j i}$.

So that in discrete form, Newton's equation of motion is expressed as

$\boldsymbol{F}_{i}-\boldsymbol{P}_{i}=\mathbb{M}_{i} \ddot{w}_{i}(t)$

where $t$ is the time, $w_{i}(t)=\left(w_{1}(t), \ldots, w_{N}(t)\right)$ is the ensemble of the position vector of $N$ points, $\boldsymbol{F}_{i}$ is the external force vector and $\boldsymbol{P}_{i}$ denotes the internal force vector; $\mathbb{M}_{i}$ refers to the mass of the point. In this paper, the velocity and displacement is updated via the Verlet-Velocity scheme [35]:

$$
\begin{array}{ll}
w_{i}(t+\Delta t) & =w_{i}(t)+\dot{w}_{i}(t) \Delta t+\frac{1}{2 \mathbb{M}_{i}}\left[\boldsymbol{F}_{i}(t)-\boldsymbol{P}_{i}(t)\right] \Delta t^{2} \\
\dot{w}_{i}(t+\Delta t) & =\dot{w}_{i}(t)+\frac{1}{2 \mathbb{M}_{i}}\left(\left[\boldsymbol{F}_{i}(t)-\boldsymbol{P}_{i}(t)\right]+\left[\boldsymbol{F}_{i}(t+\Delta t)\right.\right. \\
\left.-\boldsymbol{P}_{i}(t+\Delta t]\right) \Delta t . &
\end{array}
$$

For reasons of stability, we applied a damping term to each point

$\boldsymbol{F}_{i}^{s}=-c \dot{w}_{i}$

$\boldsymbol{F}_{i}^{S}$ representing the damping force for each point, $\dot{w}_{i}$ represents the velocity (vector) of the point, and $c$ is a damping coefficient.

The main implementation process of higher order explicit NOM for the dynamic analysis of Kirchhoff plate can be summarized as follows:

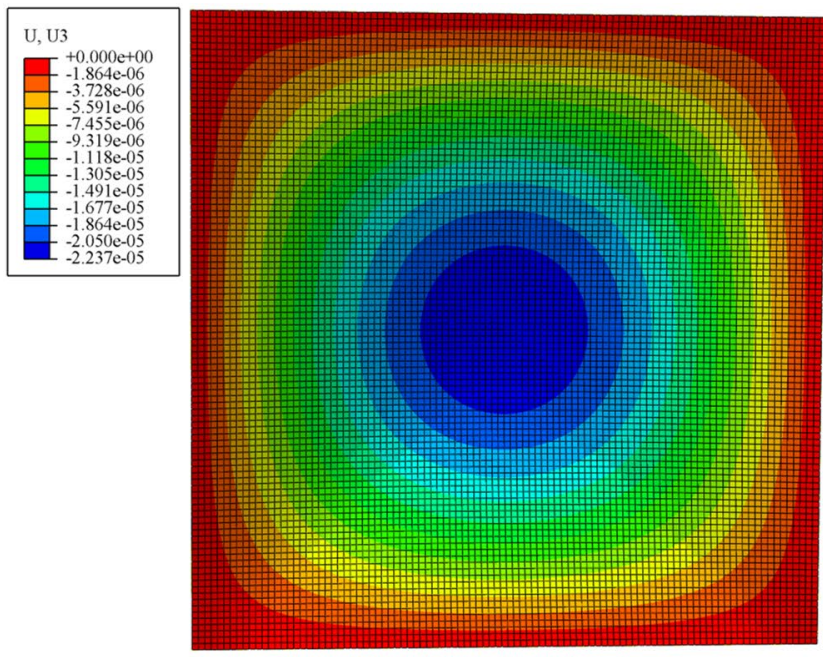

(a)
1. Discretization of the solution domain and initialization

(i) Create geometry and discretize the solution domain.

(ii) Initialize the internal force of $\boldsymbol{P}_{i}=0$ and assign values to the corresponding parameters: Young's modulus E, Poisson's ratio $v$, Density $\rho$, Number of neighbors for each point etc.

2. Calculate shape tensor

(i) For each point, calculate second, third and forth order shape tensor $\mathbf{K}_{2 i}, \mathbf{K}_{3 i}, \mathbf{K}_{4 i}$ by solving problem Eqs.8-11.

(ii) Calculate the inverse (pseudo-inverse) of the shape tensor $\mathbf{K}_{2 i}^{-1}, \mathbf{K}_{3 i}^{y} \mathbf{K}_{2 i}^{-1}, \mathbf{K}_{3 i}^{y} \mathbf{K}_{2 i}^{-1}, \mathbf{K}_{4 i}^{-1}$.

3.Calculate the nonlocal Hessian operator

For each point, calculate the nonlocal Hessian operator

$\tilde{\nabla}^{T} \tilde{\nabla} w_{i}$ by solving Eqs.17-23.

\section{Calculate the Kirchhoff plate constitutive model}

(i) Calculate Kirchhoff plate's bending stiffness and strain resultant $D_{0}, \overline{\boldsymbol{\kappa}}$ by solving Eqs.31 and 33 .

(ii) Calculate Kirchhoff plate constitutive model by solving Eq.32.

5.Calculate the Kirchhoff plate internal force between points For each neighbor point $j \in \mathcal{S}_{i}$, calculate $\boldsymbol{T}_{i i}, \boldsymbol{T}_{i i}, \boldsymbol{P}_{i j}$ by solving Eqs.51 - 52 and add $\boldsymbol{P}_{i j}$ to $\boldsymbol{P}_{i}$ and add $-\boldsymbol{P}_{i j}$ to $\boldsymbol{P}_{j}$.

\section{Applying the boundary conditions to solution}

(i) Apply the external force $\boldsymbol{F}_{i}$, damping force $\boldsymbol{F}_{i}^{s}$ and displacement boundary conditions to the specified points, according to Newton's second law $\boldsymbol{F}_{i}-\boldsymbol{P}_{i}+\boldsymbol{F}_{i}^{s}=\mathbb{M}_{i} \ddot{w}_{i}(t)$, calculate each point's acceleration $\ddot{w}_{i}$.

(ii) Update each point's velocity and displacement through the Verlet-Velocity scheme.

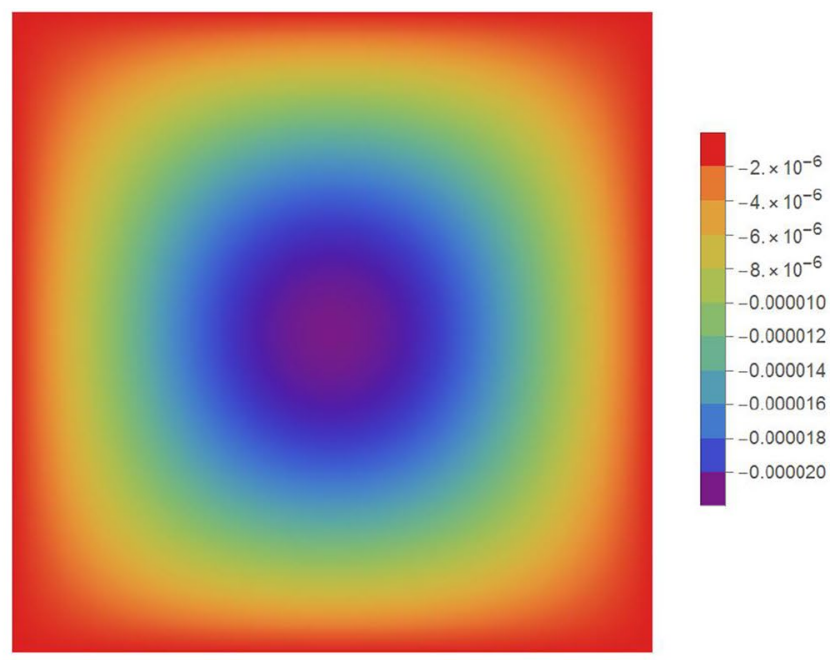

(b)

Fig. 7 Comparison of the deflection contour under uniform pressure load a ABAQUS, b nonlocal operator method 


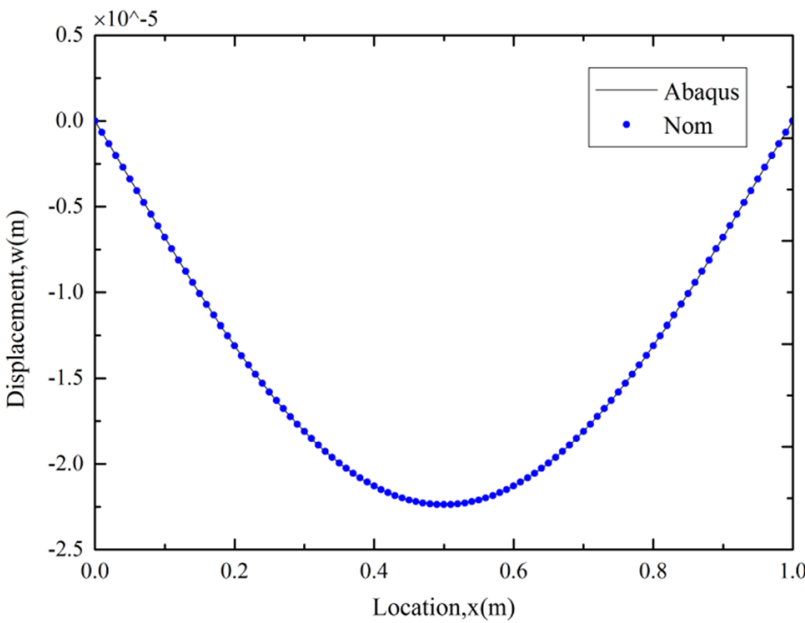

Fig. 8 Comparison of the deflection for nodes in $y=0.5$ under uniform pressure load

\section{Numerical examples}

The proposed nonlocal dynamic Kirchhoff plate formulation is implemented in Wolfram Mathematica. After verifying the accuracy of the nonlocal Hessian operator, several benchmark problems are studied and compared to results obtained by ABAQUS using the S4R plate/shell element [36].

\subsection{Verification of nonlocal Hessian operator}

Let us consider a simply supported square Kirchhoff plate with a width of $a_{0}=10 \mathrm{~m}$ and thickness $\mathrm{t}=0.01 \mathrm{~m}$. The plate is subjected to a uniform pressure of $q_{z}=100 \mathrm{~N} / \mathrm{m}^{2}$. Young's modulus and Poisson's ratio are $\mathrm{E}=30 \mathrm{GPa}$ and $v$ $=0.3$, respectively. The number of neighbors of each point is set to $n=24$. To test the accuracy of the nonlocal Hessian operator, we assume $\Upsilon=1,3$ (see Eq.58). The analytical solution of this problem is given in [37], i.e.

$$
\begin{aligned}
w & =\frac{4 q_{0} a_{0}^{4}}{\pi^{5} D_{0}} \sum_{\Upsilon=1,3, \ldots}^{\infty} \frac{1}{\Upsilon^{5}} \\
& \left(1-\frac{\alpha_{\Upsilon} \tanh \alpha_{\Upsilon}+2}{2 \cosh \alpha_{\Upsilon}} \cosh \frac{2 \alpha_{\Upsilon} y}{a_{0}}+\frac{\alpha_{\Upsilon}}{2 \cosh \alpha_{\Upsilon}} \frac{2 y}{a_{0}} \sinh \frac{2 \alpha_{\Upsilon} y}{a_{0}}\right) \\
& \sin \frac{\Upsilon \pi x}{a_{0}}
\end{aligned}
$$

with $\alpha_{\Upsilon}=\frac{\Upsilon \pi}{2}$.

We employ the quintic spline function as weight function

$\phi(\xi)=\alpha_{d}\left((1-\xi / h)_{+}^{5}-6\left(\frac{2}{3}-\xi / h\right)_{+}^{5}+15\left(\frac{1}{3}-\xi / h\right)_{+}^{5}\right)$

with $\xi=\|\xi\|, h$ is the maximum length of support, $\alpha_{d}=$ $\left(3^{5} / 40,3^{7} 7 / 478 \pi, 3^{7} / 40 \pi\right)$ with different dimensional space $d$ and $x_{+}=\max (0, x)$.

To accurately represent the relative error of the operator, we consider points at $y=0$. Figs. 5, 6 show the deflection curve of the numerical simulation $\left(\frac{\partial^{2} w}{\partial x^{2}}, \frac{\partial^{2} w}{\partial x \partial y}, \frac{\partial^{2} w}{\partial y^{2}}\right)$ compared to the analytical solution. We also check the error in the L2-norm given by

$\|w\|_{L 2}=\sqrt{\frac{\sum_{j}\left(w_{j}-w_{j}^{\text {exact }}\right) \cdot\left(w_{j}-w_{j}^{\text {exact }}\right) \Delta V_{j}}{\sum_{j} w_{j}^{\text {exact }} \cdot w_{j}^{\text {exact }} \Delta V_{j}}}$

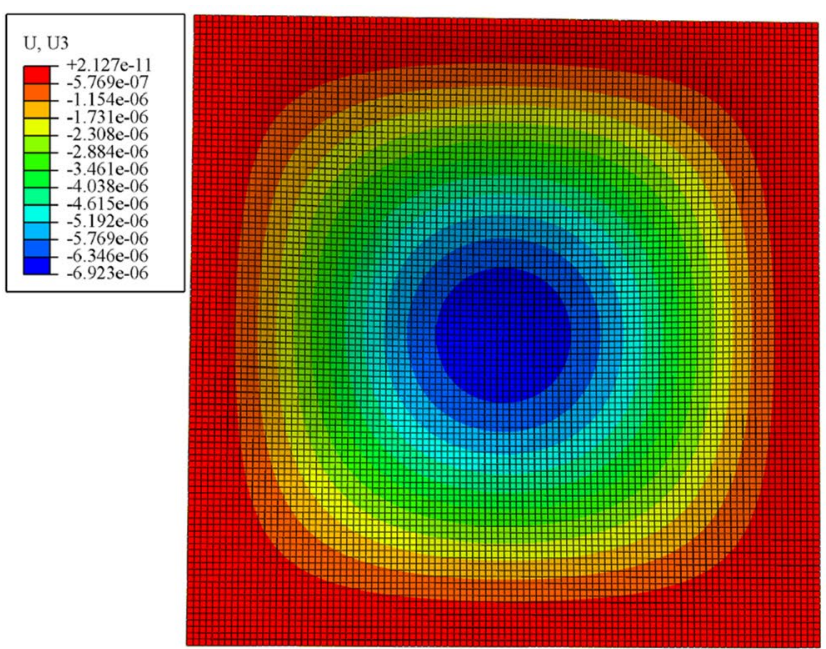

(a)

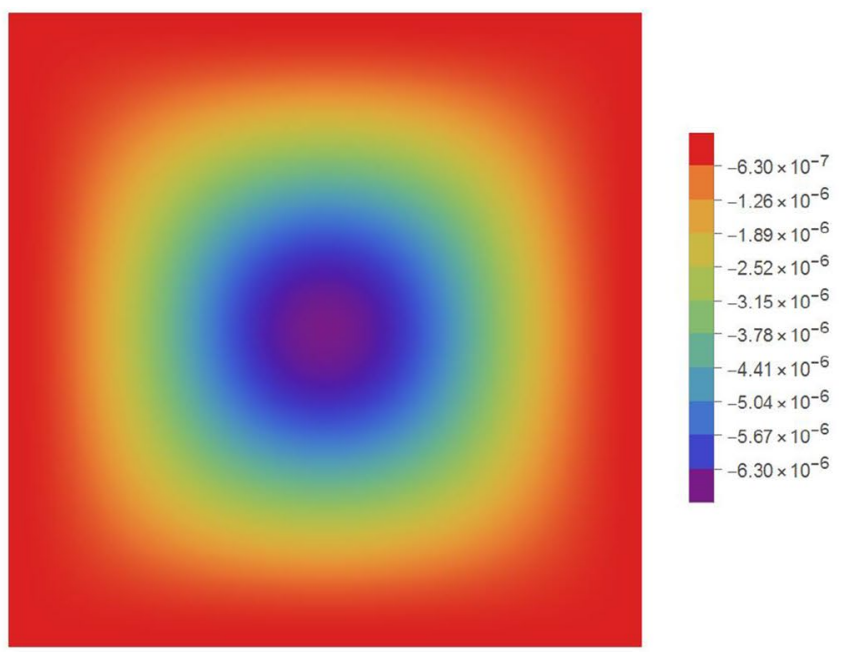

(b)

Fig. 9 Comparison of the deflection contour under uniform pressure load a ABAQUS, bnonlocal operator method 


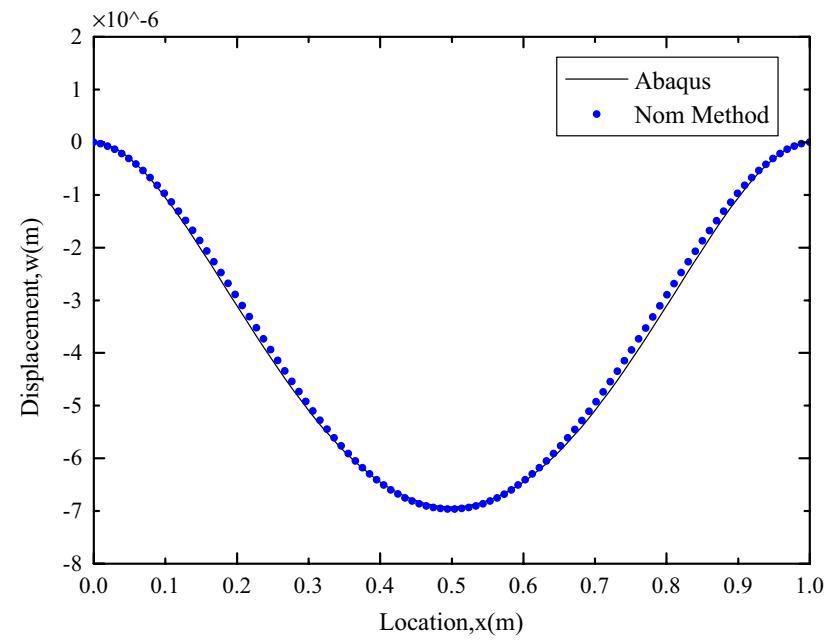

Fig. 10 Comparison of the deflection for nodes in $y=0.5$ under uniform pressure load

which is shown in Fig. 4.

\subsection{Nonlocal dynamic Kirchhoff plate formulation with simply supported boundary condition}

We now focus on a simply supported square Kirchhoff plate with a width of $a_{0}=1 \mathrm{~m}$ and thickness $\mathrm{t}=0.01 \mathrm{~m}$. Young's modulus and Poisson's ratio are $\mathrm{E}=200 \mathrm{GPa}$ and $\nu=0.3$, respectively. A uniform pressure of $q_{z}=100 \mathrm{~N} / \mathrm{m}^{2}$ is applied to the plate. The number of neighbors in the domain of influence of each point is set to $n=24$. The distance between points is selected as $\Delta x=0.01 \mathrm{~m}$ leading to 10201 points. For the ABAQUS model-as a comparison-we discretized the plate into $100 \times 100$ elements using the same input parameters.

Simply supported boundary conditions are assumed: $w(x, 0)=w(x, 1)=w(0, y)=w(1, y)=0 \quad x, y \in[0,1]$.

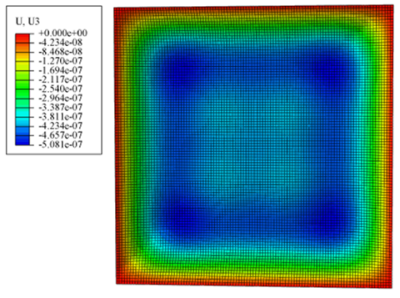

(c) ABAQUS at $\mathrm{t}=0.0005 \mathrm{~s}$

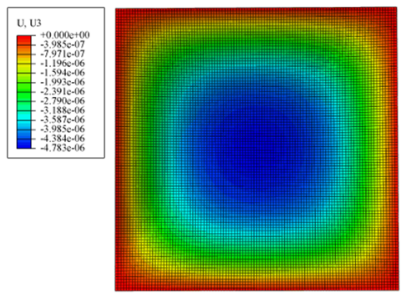

(g) ABAQUS at $\mathrm{t}=0.0015 \mathrm{~s}$
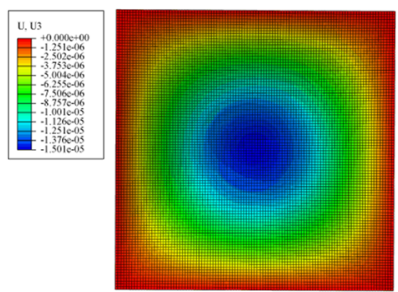

(k) ABAQUS at $\mathrm{t}=0.0025 \mathrm{~s}$

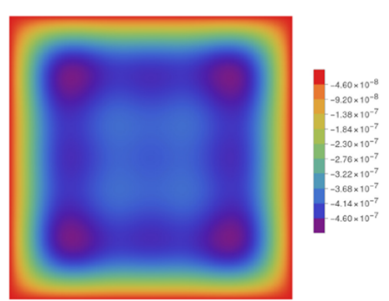

(d) NOM at t $=0.0005 \mathrm{~s}$

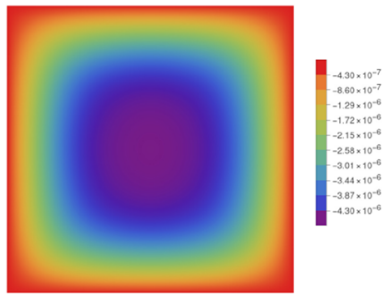

(h) $\mathrm{NOM}$ at t $=0.0015 \mathrm{~s}$

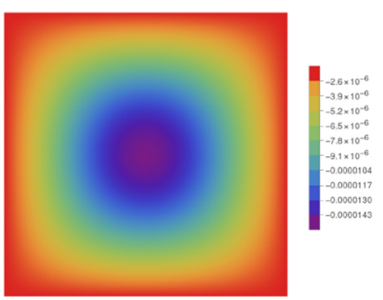

(l) $\mathrm{NOM}$ at t $=0.0025 \mathrm{~s}$

(i) ABAQUS at $\mathrm{t}=0.002 \mathrm{~s}$

(j) NOM at $\mathrm{t}=0.002 \mathrm{~s}$

Fig. 11 The evolution the deflection contour using ABAQUS and nonlocal operator method at different time 
Contour plots of the deflection can be found in Figs. 7 and 8 , respectively.

\subsection{Nonlocal dynamic Kirchhoff plate formulation with clamped boundary condition}

Next, a plate using clamped boundary conditions is studied. The geometry and the parameters of the previous section are adopted. The clamped boundary conditions are given by

$w(x, 0)=w(x, 1)=w(0, y)=w(1, y)=0$

$\frac{\partial w(0, y)}{\partial x}=\frac{\partial w(1, y)}{\partial x}=\frac{\partial w(x, 0)}{\partial y}=\frac{\partial w(x, 1)}{\partial y}=0 \quad x, y \in[0,1]$.

Contour plots of the deflection are illustrated in Figs. 9 and 10 , respectively.

\subsection{Transient test of nonlocal dynamic Kirchhoff plate formulation with simply supported boundary condition}

The last example is a simply supported plate where the damping is omitted. A uniform pressure of $q_{z}=100 \mathrm{~N} / \mathrm{m}^{2}$ is applied to the plate and the neighbors assigned to each point is set to $n=30$. All other parameters are adopted from the previous example. Figure 11 shows the deflection field at different times.

\section{Conclusions}

In this paper, a nonlocal dynamic Kirchhoff plate formulation based on a nonlocal operator method is proposed. Therefore, we derived the explicit form of the nonlocal Hessian operator taking advantage of a second order Taylor series expansion. The nonlocal operator energy functional is also derived and the relationship between local and nonlocal formulations is interpreted. In the numerical simulation section, we first verify the accuracy of the nonlocal Hessian operator for the Kirchhoff plate and compare it to analytical solutions. Subsequently, the nonlocal dynamic Kirchhoff plate formulation with different type boundary conditions (clamped and simply supported) is studied and compared to simulations obtained by ABAQUS. In the future, we intend to extend the formulation for nonlinear dynamic fracture exploiting the advantages and flexibility of NOM in this direction.

Acknowledgements The research was supported by the China Scholarship Council. The author would like to express their sincere gratitude to the editor and two anonymous reviewers for their valuable comments, which further enhanced the quality of this manuscript
Funding Open Access funding enabled and organized by Projekt DEAL.

Open Access This article is licensed under a Creative Commons Attribution 4.0 International License, which permits use, sharing, adaptation, distribution and reproduction in any medium or format, as long as you give appropriate credit to the original author(s) and the source, provide a link to the Creative Commons licence, and indicate if changes were made. The images or other third party material in this article are included in the article's Creative Commons licence, unless indicated otherwise in a credit line to the material. If material is not included in the article's Creative Commons licence and your intended use is not permitted by statutory regulation or exceeds the permitted use, you will need to obtain permission directly from the copyright holder. To view a copy of this licence, visit http://creativecommons.org/licenses/by/4.0/.

\section{References}

1. Nguyen-Thanh N, Zhou K, Zhuang X, Areias P, Nguyen-Xuan H, Bazilevs Y, Rabczuk T (2017) Isogeometric analysis of largedeformation thin shells using rht-splines for multiple-patch coupling. Comput Methods Appl Mech Eng 316:1157-1178

2. Areias P, Rabczuk T, Msekh M (2016) Phase-field analysis of finite-strain plates and shells including element subdivision. Comput Methods Appl Mech Eng 312:322-350

3. Nguyen-Thanh N, Valizadeh N, Nguyen M, Nguyen-Xuan H, Zhuang X, Areias P, Zi G, Bazilevs Y, De Lorenzis L, Rabczuk T (2015) An extended isogeometric thin shell analysis based on kirchhoff-love theory. Comput Methods Appl Mech Eng 284:265-291

4. Amiri F, Millán D, Shen Y, Rabczuk T, Arroyo M (2014) Phasefield modeling of fracture in linear thin shells. Theoret Appl Fract Mech 69:102-109

5. Areias P, Rabczuk T (2013) Finite strain fracture of plates and shells with configurational forces and edge rotations. Int J Numer Meth Eng 94:1099-1122

6. CA B (1978) The boundary element method for engineers

7. Zhang X, Liu X-H, Song K-Z, Lu M-W (2001) Least-squares collocation meshless method. Int J Numer Meth Eng 51:1089-1100

8. Greco F, Coox L, Maurin F, Desmet W (2017) Nurbs-enhanced maximum-entropy schemes. Comput Methods Appl Mech Eng 317:580-597

9. Chen J, Chen I, Chen K, Lee Y, Yeh Y (2004) A meshless method for free vibration analysis of circular and rectangular clamped plates using radial basis function. Eng Anal Boundary Elem 28:535-545

10. Roque C, Madeira J, Ferreira A (2015) Multiobjective optimization for node adaptation in the analysis of composite plates using a meshless collocation method. Eng Anal Boundary Elem 50:109-116

11. Roque C, Martins P (2015) Differential evolution optimization for the analysis of composite plates with radial basis collocation meshless method. Compos Struct 124:317-326

12. Krysl P, Belytschko T (1995) Analysis of thin plates by the element-free galerkin method. Comput Mech 17:26-35

13. Krysl P, Belytschko T (1996) Analysis of thin shells by the element-free galerkin method. Int J Solids Struct 33:3057-3080

14. Noguchi H, Kawashima T, Miyamura T (2000) Element free analyses of shell and spatial structures. Int J Numer Methods Eng 47:1215-1240

15. Ivannikov V, Tiago C, Pimenta P (2014) Meshless implementation of the geometrically exact kirchhoff-love shell theory. Int J Numer Methods Eng 100:1-39 
16. Rabczuk T, Areias P, Belytschko T (2007) A meshfree thin shell method for non-linear dynamic fracture. Int J Numer Methods Eng 72:524-548

17. Al-Tholaia MMH, Al-Gahtani HJ (2015) Rbf-based meshless method for large deflection of elastic thin plates on nonlinear foundations. Eng Anal Boundary Elem 51:146-155

18. Hussein Al-Tholaia M. M, Al-Gahtani H. J (2016) Rbf-based meshless method for large deflection of elastic thin rectangular plates with boundary conditions involving free edges, Mathematical Problems in Engineering 2016

19. Hughes TJ, Cottrell JA, Bazilevs Y (2005) Isogeometric analysis: Cad, finite elements, nurbs, exact geometry and mesh refinement. Comput Methods Appl Mech Eng 194:4135-4195

20. Cottrell JA, Hughes TJ, Bazilevs Y (2009) Isogeometric analysis: toward integration of CAD and FEA. John Wiley \& Sons

21. Kiendl J, Bletzinger K-U, Linhard J, Wüchner R (2009) Isogeometric shell analysis with kirchhoff-love elements. Comput Methods Appl Mech Eng 198:3902-3914

22. Riffnaller-Schiefer A, Augsdörfer UH, Fellner DW (2016) Isogeometric shell analysis with nurbs compatible subdivision surfaces. Appl Math Comput 272:139-147

23. Benson D, Bazilevs Y, Hsu M-C, Hughes T (2010) Isogeometric shell analysis: the reissner-mindlin shell. Comput Methods Appl Mech Eng 199:276-289

24. Thai CH, Nguyen-Xuan H, Bordas SPA, Nguyen-Thanh N, Rabczuk T (2015) Isogeometric analysis of laminated composite plates using the higher-order shear deformation theory. Mech Adv Mater Struct 22:451-469

25. Li K, Wu D, Gao W (2019) Spectral stochastic isogeometric analysis for linear stability analysis of plate. Comput Methods Appl Mech Eng 352:1-31

26. Eringen A. C (2002)Nonlocal continuum field theories, Springer Science \& Business Media

27. Silling S (2000) Reformulation of elasticity theory for discontinuities and long-range forces. J Mech Phys Solids 48:175-209
28. Bažant ZP, Jirásek M (2002) Nonlocal integral formulations of plasticity and damage: survey of progress. J Eng Mech 128:1119-1149

29. Gunzburger M, Lehoucq RB (2010) A nonlocal vector calculus with application to nonlocal boundary value problems. Multiscale Model Simul 8:1581-1598

30. Ren H, Zhuang X, Rabczuk T (2020) A nonlocal operator method for solving partial differential equations. Comput Methods Appl Mech Eng 358:112621

31. Rabczuk T, Ren H, Zhuang X (2019) A nonlocal operator method for partial differential equations with application to electromagnetic waveguide problem. Comput Mater Continua 59 (2019). Nr. 1

32. Samaniego E, Anitescu C, Goswami S, Nguyen-Thanh VM, Guo H, Hamdia K, Zhuang X, Rabczuk T (2020) An energy approach to the solution of partial differential equations in computational mechanics via machine learning: Concepts, implementation and applications. Comput Methods Appl Mech Eng 362:112790

33. Guo H, Zhuang X, Rabczuk T (2021) A deep collocation method for the bending analysis of kirchhoff plate, arXiv preprint arXiv: 2102.02617

34. Ren H, Zhuang X, Rabczuk T (2020) A higher order nonlocal operator method for solving partial differential equations. Comput Methods Appl Mech Eng 367:113132

35. Verlet L (1967) Computer" experiments" on classical fluids. i. thermodynamical properties of lennard-jones molecules. Phys Rev 15998

36. Hibbett Karlsson (1998) Sorensen, ABAQUS/standard: User's Manual, vol 1. Karlsson \& Sorensen, Hibbitt

37. Timoshenko S. P, Woinowsky-Krieger S (1959)Theory of plates and shells, McGraw-hill

Publisher's Note Springer Nature remains neutral with regard to jurisdictional claims in published maps and institutional affiliations. 